DOI: http://dx.doi.org/10.15517/rce.v33i1.19995

\title{
CRITERIOS PARA LA IDENTIFICACIÓN Y DEFINICIÓN DE TERRITORIOS CON POTENCIAL DE DESARROLLO PRODUCTIVO PARA EL ESTABLECIMIENTO DE ZONAS ECONÓMICAS ESPECIALES DE DESARROLLO (ZEED) EN COSTA RICA
}

\author{
Rafael Arias Ramírez ${ }^{1}$ \\ Leonardo Sánchez Hernández ${ }^{2}$ \\ Luis Vargas Montoya ${ }^{3}$ \\ Oscar Agüero Rodríguez ${ }^{4}$
}

Recibido: 02/02/2015

Aprobado: 29/06/2015

\begin{abstract}
RESUMEN
Este estudio tiene como propósito identificar, a partir de una serie de variables e indicadores, territorios que cuenten con condiciones propicias para un desarrollo productivo endógeno, basado en la dotación de recursos y en una política productiva a nivel nacional $y$ local que lo promueva. Se construye un índice cantonal de potencial productivo alrededor de tres ejes (productivo, institucional e infraestructura), al que se le aplica el método de análisis de conglomerados. Los resultados del estudio no solo son novedosos para el país; sino que, además, contribuyen con la construcción de una matriz de variables e indicadores que proveen criterios empíricamente validados para identificar y definir ZEED para Costa Rica.
\end{abstract}

PALABRAS CLAVE: GRAN ÁREA METROPOLITANA, DESARROLLO ENDÓGENO, NÚCLEOS ENDÓGENOS, ÍNDICE DE POTENCIAL PRODUCTIVO CANTONAL, ANÁLISIS DE CONGLOMERADOS, COMPETITIVIDAD TERRITORIAL.

\section{ABSTRACT}

This study is aimed to identify, based on a series of variables and indicators, territories that have characteristics to propitiate an endogenous development, from the basis of

1 Universidad de Costa Rica, Instituto de Investigaciones en Ciencias Económicas, Código Postal 11501-2060, Costa Rica; rafael.arias.ramirez@una.ac Rica; joseleonardo.sanchez@ucr.ac.cr

3 Universidad de Costa Rica, Instituto de Investigaciones en Ciencias Económicas, Código Postal 11501-2060, Costa Rica; lvargas22@gmail.com

4 Universidad de Costa Rica, Instituto de Investigaciones en Ciencias Económicas, Código Postal 11501-2060, Costa Rica; osagrodriguez@gmail.com 
the resource endowment they have, along with a productive policy, at the national and local level to promote it. A county's index of productive potential is built around three components (productive, infrastructure and institutions); then a cluster analysis is applied. The outcomes of the study are not only new for the country, also they make a contribution with the elaboration of a matrix of variables and indicators, which provide criteria, empirically validated, to identify and define Special Economic Zones of Development (SEZD) for Costa Rica.

KEYWORDS: GREAT METROPOLITAN AREA, ENDOGENOUS DEVELOPMENT, ENDOGENOUS CORES, PRODUCTIVE POTENTIAL INDEX, ANALYSIS OF CONGLOMERATES, TERRITORIAL COMPETITIVENESS.

\section{INTRODUCCIÓN}

La dinámica económica y productiva de Costa Rica ha estado concentrada y centralizada en la Gran Área Metropolitana (GAM). Los altos niveles de aglomeración de las actividades productivas, institucionales y administrativas han convertido a la GAM en la zona económica de desarrollo más importante del país. Esto se confirma en la dinámica de los sectores productivos, especialmente secundario y terciario, como en el desarrollo del aparato político-administrativo, con una presencia preponderante en la GAM. Como consecuencia de lo anterior, se ha dado un uso intensivo y poco planificado del territorio y del uso del suelo, tanto para promover las actividades productivas, especialmente del sector industrial, como también para albergar una población en continuo crecimiento.

Lo anterior se refleja en la configuración de los corredores industriales que si bien es cierto su expansión sigue atendiendo a las restricciones geográficas, su localización ha tendido a desplazarse al margen de las zonas de mayor urbanización. Estas últimas se han incrementado fuera de las áreas tradicionales generando nuevos polos de desarrollo dentro de la GAM, especialmente en las Áreas Metropolitanas (AM) de Heredia, Alajuela y Cartago. Es interesante también señalar que los cambios en la geografía económica de la GAM, ahora más apegados a criterios de rentabilidad, estén teniendo en cuenta las ventajas competitivas territoriales y de dotación de recursos naturales $y$ humanos, como determinantes del clima de negocios y de las decisiones de inversión del capital, tanto nacional como internacional (Arias y Sánchez, 2012).

El desarrollo, localización y expansión de las actividades productivas, medidas a través del empleo dentro de la GAM, muestran un patrón que no necesariamente obedece a estrategias de localización espacial, producto de las economías de escala generadas por la aglomeración y la existencia de instituciones $y$ de mecanismos institucionales eficientes para la promoción, atracción y consolidación de inversiones con una alta capacidad competitiva y de cooperación entre ellas. Asimismo, los vacíos en cuanto a un adecuado ordenamiento urbano, gestión del territorio y sus recursos, aunado al congestionamiento vial han conducido a una elevada concentración y centralización de las actividades productivas en la GAM, condicionando el desarrollo de otras zonas geográficas del país y generando externalidades negativas relacionadas con el uso intensivo de los recursos naturales (suelo y recurso hídrico), la contaminación ambiental y el aumento de los costos de transacción para los agentes económicos (Arias y Sánchez, 2012).

De esta manera, en la medida en que el proceso de expansión urbanística de las ciudades fue encareciendo los terrenos y generando restricciones para una adecuada movilización de los bienes y servicios, las industrias comenzaron un proceso de emigración hacia otros territorios, aunque siempre dentro de la misma GAM (proceso de movilización intra-GAM).

En los próximos años la demanda de uso de suelo industrial dentro de los cantones de la GAM dependerá de la dinámica de la actividad industrial y de la capacidad del país para seguir atrayendo 
inversión extranjera directa, lo cual pasa por resolver las limitaciones que actualmente se presentan en infraestructura, como la congestión de la red vial, el aumento significativo en precios de terrenos en algunas zonas, la disponibilidad y abastecimiento continuo de insumos cerca de las zonas industriales establecidas, la tramitología para iniciar negocios y desarrollar emprendimientos, entre otros. Igualmente, el futuro de una actividad industrial más dinámica y competitiva en la GAM dependerá de una transformación productiva que esté más articulada, tanto sectorialmente como con el desarrollo de otras zonas económicas de desarrollo en que involucren otras regiones del país.

Relacionado con lo anterior, el país necesita potenciar, a partir de la dinámica de desarrollo de la GAM, otras zonas económicas de desarrollo en otros territorios con características estratégicas de localización y dotación de recursos endógenos complementarios al desarrollo de la GAM $y$ del país en general.

Este estudio tiene como propósito identificar, a partir de una serie de variables e indicadores, territorios que cuentan con una serie de condiciones que pueden propiciar un desarrollo productivo endógeno, basado en la dotación de recursos con los que se cuentan y en una política productiva a nivel nacional y local que los promueva. De tal manera, en las siguientes páginas se explican los procedimientos metodológicos que se han seguido para obtener el índice de potencial productivo cantonal y el análisis de conglomerado cantonal. Los resultados del estudio no solo son novedosos para el país, sino que, además, contribuyen con la construcción de una matriz de indicadores y variables que proveen criterios empíricamente validados para identificar y definir ZEED en Costa Rica ${ }^{5}$.

\section{MARCO TEÓRICO - METODOLÓGICO}

En esta sección se presenta el fundamento teórico-metodológico para la identificación y definición de ZEED en las distintas regiones de Costa Rica con alto potencial de desarrollo productivo. Para ello, acorde con la revisión bibliográfica relevante en torno al tema, tanto a nivel nacional como internacional, así como con la experiencia de investigaciones previas realizadas en el IICE ${ }^{6}$, se identifican una serie de indicadores relevantes para el objeto de la presente investigación. Estos se clasifican en tres grandes ejes: 1) especialización productiva, 2) infraestructura e 3) institucional.

A partir de los indicadores sugeridos por la literatura y de acuerdo con la disponibilidad de datos para el caso costarricense, se identifican un conjunto de variables que posibilitan su medición en forma aproximada. Para ello, se define al cantón como la unidad estadística territorial de mayor desagregación posible, en la que se cuenta con información estadística para la aproximación de los indicadores relevantes ${ }^{7}$.

De las opciones metodológicas consideradas, se opta por la construcción de un índice conjunto de las variables relevantes para el estudio de ZEED en una primera etapa (Arias et al., 2015). Posteriormente, en una segunda etapa, se elige el método multivariado de análisis de conglomerados para la evaluación conjunta de los valores obtenidos en el índice. Estas dos aplicaciones metodológi-

$5 \quad$ Es importante destacar que, al ser la presente investigación pionera en cuanto a la identificación y definición de ZEED, el procedimiento metodológico y variables seleccionadas para una primera etapa del proyecto de investigación son sujeto de ajuste en una segunda etapa; para ello, se realizarán ejercicios de validación con los datos obtenidos y se considerará el criterio de expertos

El IICE tiene ya cerca de 10 años de experiencia en investigaciones en el campo de la economía regional, las cuales se han llevado a cabo a lo largo del territorio nacional: Turrialba, Gran Puntarenas, Pérez Zeledón, Gran Área Metropolitana y la Región Chorotega. Para más información se puede consultar la página del Instituto.

$7 \quad$ Costa Rica está compuesta por ochenta y un cantones, distribuidos en forma proporcional al tamaño de la población de las siete provincias que componen el país. De estos, 31 conformar la GAM (23 en su totalidad y 8 parcialmente). 
cas se seleccionan al ser los métodos, que a la luz de los datos disponibles, se constituyen como los más apropiados para cumplir con el objetivo de la investigación (De la Fuente, 2011).

Una vez definido el procedimiento de análisis metodológico a llevar a cabo, se realiza la selección de las variables que posibilitan la aproximación de los indicadores relevantes para el estudio de las ZEED (cuadro 1$)^{8}$.

\section{CUADRO 1 \\ VARIABLES DEL ÍNDICE CANTONAL PARA LA IDENTIFICACIÓN Y DEFINICIÓN DE REGIONES CON ALTO POTENCIAL DE DESARROLLO PRODUCTIVO PARA EL ESTABLECIMIENTO DE ZEED ${ }^{9}$}

\begin{tabular}{|c|c|c|}
\hline Especialización productiva & Infraestructura & Institucional \\
\hline $\begin{array}{l}\text { Generación de empleo total } \\
\text { (volumen) }\end{array}$ & Tenencia y estado del plan regulador & $\begin{array}{l}\text { Porcentaje de participación en elecciones } \\
\text { presidenciales }\end{array}$ \\
\hline \multirow{2}{*}{$\begin{array}{l}\text { Porcentaje de empleo real con } \\
\text { secundaria completa o más } \\
\text { (calidad) }\end{array}$} & Gestión vial & $\begin{array}{l}\text { Porcentaje de participación en elecciones } \\
\text { municipales }\end{array}$ \\
\hline & $\begin{array}{l}\text { Distancia respecto al puerto más } \\
\text { cercano }(-1)^{*}\end{array}$ & $\begin{array}{l}\text { Índice de Seguridad Cuidadana Cantonal } \\
\text { (ISCc) }\end{array}$ \\
\hline Porcentaje de empleo proveniente & Distancia respecto a aeropuertos $(-1)$ & Planificación \\
\hline \multirow[t]{8}{*}{ de otras localidades (movilidad) } & $\begin{array}{l}\text { Distancia respecto a la frontera más } \\
\text { cercana }(-1)\end{array}$ & Gestión financiera \\
\hline & Penetración de banda ancha & Recursos humanos \\
\hline & Depósito y tratamiento de residuos & Control interno \\
\hline & Aseo de vías & $\begin{array}{l}\text { Instituciones financieras por cada } 1000 \\
\text { habitantes }\end{array}$ \\
\hline & Recolección de residuos & Ebais por cada 1000 habitantes \\
\hline & $\begin{array}{l}\text { Precio por metro cuadrado según } \\
\text { actividad (-1) }\end{array}$ & $\begin{array}{l}\text { Presencia de instituciones para } \\
\text { universitarias }\end{array}$ \\
\hline & Riesgo ante eventos extremos $(-1)$ & Acceso a formación del INA \\
\hline & & $\begin{array}{l}\text { Colegios técnicos por cada } 10000 \\
\text { habitantes }\end{array}$ \\
\hline
\end{tabular}

* El (-1) denota una relación inversa entre la variable y su aporte al eje, por ello, los valores originales se transforman para incluirse en el índice.

A continuación se presenta la descripción detallada de las variables que aproximan cada indicador según el eje que componen.

\section{- $\quad$ Eje especialización productiva}

Un primer eje que engloba una serie de indicadores para evaluar el potencial de una región para constituirse como una ZEED es el de especialización productiva. Este tiene como objetivos analizar la estructura y especialización productiva de cada cantón, caracterizar el perfil de la fuerza

8 Es importante destacar que los criterios de mayor relevancia para seleccionar estas variables son la disponibilidad de datos para la totalidad de cantones del país, su capacidad para discriminar los cantones y la posibilidad de que puedan ser replicadas $y$ actualizadas en estudios posteriores.

9 Resulta importante aclarar que aunque se cuenta con información para varios años (inclusive al cierre del 2014) en algunas de estas variables, en atención a que el último Censo nacional de población se realiza en el 2011, en el índice únicamente se incluyen los valores de las variables más próximas a este año. 
laboral a nivel cantonal y, por último, categorizar los cantones según su grado de especialización productiva. Dentro de este eje se seleccionan los siguientes indicadores: i) generación de empleo, ii) calificación del empleo y iii) procedencia de los trabajadores.

\section{- Generación de empleo}

La importancia de esta variable radica, como señala Ishida (2009), en que los territorios con una alta densidad de mano de obra se constituyen como potenciales Zonas Económicas Especiales. Así, lo que se busca es identificar el peso de empleo real relativo del cantón con respecto al total del país y determinar cuáles son los cantones con mayor densidad de mano de obra.

Esta variable se construye a partir de los datos extraídos del Censo 2011, realizado por el Instituto Nacional de Estadística y Censos (INEC). Para ello, se identifica la densidad de empleo real por cantón $\left(D_{\text {empleo real }}\right)$, a partir de la cantidad de trabajadores del cantón $\left(L_{\text {cantón }}\right)$ y el total del país $\left(L_{\text {pais }}\right)$, como sigue:

$$
D_{\text {empleo real }}=\frac{L_{\text {cantón }}}{L_{\text {pais }}}
$$

Los resultados van a oscilar entre $0 \leq D_{-} \_(e m p l e o \text { real }) \leq 1$, donde 0 es la menor densidad posible de trabajadores dentro de un cantón y 1 representa el caso hipotético de que un cantón concentre la totalidad del empleo del país.

\section{- Calificación del empleo}

Esta variable se justifica a partir del término "territorios globales", el cual Sassen (1991) define como aquellas zonas que disponen de algunos factores competitivos como infraestructura, soporte tecnológico apropiado, mano de obra calificada, entre otros.

En esa línea, se mide el nivel de calificación de la fuerza laboral del cantón, a partir de la proporción de trabajadores con educación técnica, parauniversitaria ${ }^{10}$ y universitaria, como sigue:

$$
C E_{i}=\frac{X_{i}+Y_{i}+Z_{i}}{E_{i}}
$$

donde:

$C E_{i}$ : Proporción de empleo calificado en el cantón $i$

$X_{i}$ : Trabajadores con educación secundaria técnica en el cantón $i$

$Y_{i}$ : Trabajadores con educación parauniversitaria en el cantón $i$

$Z_{i}$ : Trabajadores con educación universitaria en el cantón $i$

"E" : Total de trabajadores del cantón $i$

Esta variable puede oscilar entre un valor 0 en caso de que el cantón no cuente con trabajadores calificados y un valor máximo de 1 en el que todos los trabajadores del cantón sean calificados.

10 Puerto Moín y Puerto Caldera son las dos ventanas marítimas de mayor relevancia en el país, las cuales se ubican en el Mar Caribe y en el Océano Pacífico, respectivamente 


\section{- Procedencia de los trabajadores}

Como señalan Boix y Galletto (2005), quienes adoptan la definición de los sistemas locales de trabajo ${ }^{11}$ como zonas que son relativamente independientes en cuanto a los desplazamientos diarios de trabajadores desde su casas a su lugar de trabajo, se entiende que las zonas con menor porcentaje de desplazamiento de fuerza laboral serán aquellas que son más independientes $y$, por ende, satisfacen en mayor medida su demanda laboral. No obstante, ante la necesidad de atraer empleo de otras localidades, como un indicador de conectividad, resulta importante determinar en qué medida este desplazamiento es posible.

La variable se obtiene de la diferencia entre el total de trabajadores del cantón y los trabajadores del cantón que residen en el mismo, cuyo resultado es la cantidad de trabajadores provenientes de otros cantones. Luego se expresa en términos relativos.

Dentro de los valores esperados se podría obtener un valor mínimo de $0 \%$, el cual indica independencia del cantón en cuanto a desplazamiento de trabajadores y un máximo de $100 \%$, que representa dependencia total.

\section{- Eje infraestructura}

La disponibilidad de infraestructura de transportes, comunicación, edificaciones, entre otros; así como la presencia de estándares medio ambientales aceptables, se constituyen en generadores de ventajas competitivas estáticas de los territorios (Cabrero et ál., 2003). Sumado a ello, los lineamientos en torno al desarrollo de infraestructura y la vulnerabilidad ante amenazas naturales, son elementos claves para el desarrollo de la actividad económica de una localidad. Por ello, un segundo eje que incorpora la presente investigación es el de infraestructura, el cual agrupa un conjunto de indicadores propuestos por la literatura y cuya medición se aproxima a través de las variables que se describen a continuación.

\section{- Tenencia y estado del plan regulador}

Un primer aspecto a tener en consideración respecto del desarrollo de infraestructura en una localidad, es el relativo a las restricciones reglamentarias de ordenamiento para su desarrollo. Así, la existencia de un plan regulador es uno de los elementos fundamentales para brindar seguridad jurídica a las empresas que pretendan instalarse en un territorio, las cuales deben contar con reglas claras del juego para sus expectativas de inversión. En esa línea, una de las variables que se evalúa es la presencia y estado de un plan regulador en los cantones del país, cuyas categorías se describen en el siguiente cuadro:

CUADRO 2

VALORES DE CATEGORIZACIÓN SEGÚN PRESENCIA Y ESTADO DEL PLAN REGULADOR

\begin{tabular}{lc}
\hline Categoría & Valor \\
\hline No vigente & 0 \\
Vigente desactualizado & 0,33 \\
Vigente en proceso de actualización & 0,67 \\
Vigente actualizado & 1 \\
\hline
\end{tabular}

11 Los datos de las siguientes dos variables se extraen de la misma fuente. 


\section{- Gestión vial}

Esta variable evalúa el grado de cumplimiento de metas según el presupuesto destinado a este sector, en cuanto al alcance de las metas programadas en el plan operativo anual, la ejecución de recursos y la condición de la superficie de ruedo de la red vial cantonal.

Los datos se extraen del Índice de Gestión Municipal (IGM) ${ }^{12}$, elaborado anualmente por la Contraloría General de la República (CGR), el cual evalúa el desempeño de los gobiernos locales (municipalidades) de cada cantón en diferentes áreas.

Los valores esperados van a oscilar entre 0 , que corresponde al caso de cantones con un incumplimiento total de las metas según cronograma y un valor máximo de 1, que refleja el cumplimiento del $100 \%$ de las metas programadas ${ }^{13}$.

\section{- Distancia y tiempo de viaje}

Dentro de los indicadores de relevancia a la hora de evaluar un territorio como un candidato potencial a ZEED, se encuentran los que Tong De et ál. (2010) denominan componentes locacionales, donde se ubica la distancia de una ciudad respecto a infraestructura básica para el desarrollo de la actividad productiva. Como parte de esta infraestructura básica se identifican la facilidad de acceso a puertos, aeropuertos y comunicaciones globales, como variables clave para la localización de nuevas industrias (Markussen, Hall y Glassmeier; 2000). Para medir el acceso relativo de los cantones a la infraestructura logística más importante, se utilizan las siguientes variables:

Distancia respecto al puerto más cercano $(-1)$

Una primera variable de interés es la distancia que presenta un cantón respecto al puerto más cercano. A mayor cercanía, menores serán los costos de transacción que una industria localizada en el cantón deba incurrir para llevar a cabo su actividad de negocios, especialmente si está vinculada con el mercado internacional. Así, con base en los datos de distancias en km de los cantones respecto a Puerto Moín y Puerto Caldera ${ }^{14}$ del Programa de Investigación en Desarrollo Urbano Sostenible (ProDUS) de la Universidad de Costa Rica (UCR) al año $2014^{15}$, se construye la siguiente variable:

12 El índice contempla 89 municipalidades, lo cual es mayor al número de cantones que es de 81; esto es debido a la lejanía que presentan ciertos distritos respecto a la cabecera de cantón, por lo que algunos cantones cuentan con más de un gobierno local. Así, se realiza el ajuste del IGM al asignar el mismo peso a la totalidad de los distritos que componen el cantón y, posteriormente, se pondera la calificación de los gobiernos locales distritales con la calificación del cantón al que pertenecen (este mismo ajuste se realiza en las demás variables del IGM que se incluyen en la presente investigación). El procedimiento se detalla en la siguiente ecuación:

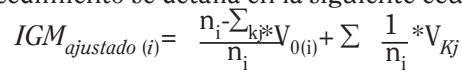

donde:

$n_{i}:$ cantidad total de distritos en el cantón $i$.

$k_{i}$ : Consejos municipales de distrito en el cantón $i$.

$\mathrm{V}_{0(\mathrm{i})}$ : Valor inicial indicado por el IGM en el cantón $i$.

$\mathrm{V}_{\mathrm{kj}}$ : Valor indicado para cada consejo municipal del distrito $j$

13 Valga aclarar que algunos cantones podrán presentar calificaciones de $0 \%$ en caso de que no hayan entregado la información que se incluye en el IGM o al no contar con el desempeño mínimo del indicador. No obstante, para efectos de la presente aplicación metodológica, no se hace diferencia entre ambas posibilidades.

Puerto Moín y Puerto Caldera son las dos ventanas marítimas de mayor relevancia en el país, las cuales se ubican en el Mar Caribe y en el Océano Pacífico, respectivamente.

Los datos de las siguientes dos variables se extraen de la misma fuente. 
donde:

$$
\text { Dpuerto }_{i j}=\frac{\text { ppuerto }_{(\max (j)}-D_{i j}}{\text { Dpuerto }_{\max (\mathrm{j})}}
$$

Dpuerto $_{\mathrm{ij}}=$ Valor de distancia del cantón i respecto al puerto $j$

Dpuerto $_{\max (j)}$ : Distancia en Km del cantón que se encuentra más lejos del puerto $j$ $\mathrm{D}_{\mathrm{ij}}$ : Distancia en Km del cantón $i$ respecto al puerto $j$

Posteriormente, partiendo del hecho de que la cercanía del cantón con algún puerto es una condición favorable para el flujo de bienes y servicios, se selecciona el valor máximo obtenido por el cantón i tras aplicar la ecuación anterior. Se espera que esta variable oscile entre un valor mínimo de cero y un valor máximo que corresponde al cantón con menor distancia a un puerto.

\section{- Distancia respecto a aeropuertos (-1)}

Entre más cerca de un cantón esté un aeropuerto, menores serán los costos de transacción en los que las empresas incurren en su proceso de producción y circulación de capital, especialmente si son actividades vinculadas con el mercado internacional. Así, partiendo de los datos de distancias en $\mathrm{km}$ de los cantones respecto al Aeropuerto Internacional Juan Santamaría, se construye la siguiente variable:

$$
\text { Dapuerto }_{i j}=\frac{\text { Dapuerto }_{(\max (j)}-D_{i j}}{\text { Dapuerto }_{\max (\mathrm{j})}}
$$

donde:

Daeropuerto $_{\mathrm{i}}=$ Valor de distancia respecto a aeropuerto Juan Santamaría para el cantón $i$ Daeropuerto $_{\max }$ : Distancia en Km del cantón que se encuentra más lejos del aeropuerto Juan Santa María

$\mathrm{D}_{\mathrm{i}}$ : Distancia en Km del cantón $i$ respecto al aeropuerto Juan Santamaría

Se espera que la variable oscile entre un valor mínimo cero y un valor de máximo asociado al cantón que se encuentra más cerca de Aeropuerto.

\section{- Distancia respecto a la frontera más cercana (-1)}

Se supone que cuanto más cerca esté el cantón de las fronteras terrestres del territorio nacional, menor es el costo de transacción que debe asumir una industria en su producción y circulación de capital, especialmente si son actividades vinculadas con el mercado regional centroamericano. Por ello, con base en los datos de distancias en $\mathrm{km}$ de los cantones respecto a las fronteras de Peñas Blancas y Paso Canoas, se construye la variable:

$$
\text { Dfronteraij }=\frac{\text { Dfrontera }}{(\max (j)}_{\text {Dfrontera }_{\max (\mathrm{j})}}-D_{i j}
$$

donde:

Dfrontera $_{i j}=$ Valor obtenido por el cantón $i$ respecto a la frontera $j$

Dfrontera $_{\max (\mathrm{j})}$ : Distancia en Km del cantón que se encuentra más lejos de la frontera $j$

$\mathrm{Di}_{\mathrm{i}}$ : Distancia en Km del cantón $i$ respecto a la frontera $j$ 
Posteriormente, partiendo del hecho de que la cercanía de un cantón, al menos, a una de las fronteras le permite una mayor rapidez en el traslado de bienes, se selecciona el valor máximo obtenido por el cantón $i$ tras aplicar la ecuación anterior. Se esperan valores entre un valor mínimo de cero y un valor máximo (menor o igual a uno) para el cantón más cerca de la frontera.

\section{- Telecomunicaciones}

Hoy más que nunca la economía se ha globalizado y las nuevas tecnologías de la información $y$ las telecomunicaciones (TICs) se han convertido en factores determinantes de la competitividad de los países. Como señalan Kayser et ál. (2014), uno de los indicadores de mayor relevancia dentro de las telecomunicaciones es el acceso a Internet de banda ancha, el cual, empíricamente, se ha demostrado que produce importantes efectos sobre la economía, siendo uno de estos el incremento en la productividad de las industrias.

\section{Penetración de banda ancha}

Para aproximar dicha variable, se parte de los datos de la "VIII medición de la penetración de Internet de banda ancha en Costa Rica" del Barómetro de Cisco. Esta corresponde al porcentaje de personas del cantón i con acceso a Internet de banda ancha. Dicha variable oscila entre un valor mínimo de $0 \%$, en caso de que los pobladores del cantón no cuenten con acceso a Internet de banda ancha, $y$ un valor máximo de $100 \%$, en el que la totalidad de los habitantes del cantón cuentan con acceso a Internet de banda ancha.

\section{- Gestión ambiental}

Se mide a partir del IGM, por medio de tres variables:

1. recolección de residuos

2. depósito y tratamiento de residuos y

3. aseo de vías y sitios públicos.

Se evalúa si las municipalidades ofrecen los servicios citados, si la tasa que se cobra para su prestación se encuentra actualizada, la medición de la proporción de los ingresos en relación con los gastos de operación de los servicios y sus niveles de cobertura.

Estas tres variables se consideran en el presente índice y podrán oscilar entre un valor mínimo de $0 \%$ y un valor máximo del $100 \%$, de acuerdo con el grado de cumplimiento de la programación de metas.

\section{- Valor de la tierra}

De acuerdo con Tong De et ál. (2010), uno de los elementos económicos de importancia para el desarrollo de una ZEED es la renta de la tierra. Por ello, en la presente investigación se incorpora una variable que busca aproximar dicho indicador. 


\section{Precio por metro cuadrado según actividad (-1)}

Partiendo de los datos del precio del $\mathrm{m} 2$ de construcción según actividad (residencia, comercio, servicio, industria y agropecuario), generados por ProDUS para el año 2014 y, en razón de que a mayor precio por $\mathrm{m}^{2}$ de construcción, la inversión que debe realizar una empresa para localizarse en un cantón es más alta, la variable se construye como sigue:

donde:

$$
\text { Valor mi2 }=\frac{P m^{2}{ }_{\max }-P m^{2}}{P m^{2}{ }_{\text {max }}}
$$

Valor $\mathrm{m}^{2}=$ Valor de la variable precio por $\mathrm{m}^{2}$ en el cantón $i$

$\mathrm{Pm}^{2}{ }_{\max }$ : Precio máximo por $\mathrm{m}^{2}$ según cantón

$\mathrm{Pm}^{2}$ : Precio por $\mathrm{m}^{2}$ en el cantón $i$

Tras su aplicación, los valores oscilarán entre un mínimo de cero y el valor máximo que obtendrá el cantón que cuente con el menor precio por $\mathrm{m}^{2}$.

\section{- Amenazas naturales}

Otro de los indicadores relevantes a la hora de valorar la localización de industrias en un territorio, es la vulnerabilidad que este presenta ante amenazas naturales; esto por cuanto las empresas no solo deben realizar una inversión inicial mayor en infraestructura, sino también prever el posible efecto en costos de un evento natural sobre su estructura.

\section{Riesgo ante eventos extremos (-1)}

Para la construcción de esta variable se parte de los datos de zonas de riesgo ante eventos extremos secos y lluviosos de ProDUS (2013), que clasifican los cantones como zonas de alto, medio y bajo riesgo según tipo seco y lluvioso, asignándoles un valor de 3, 2 y 1 respectivamente.

En esa línea, se construye una variable categórica que pondera el valor obtenido por el cantón según tipo, con lo que se obtienen los siguientes valores de referencia:

CUADRO 3

VALORES DE REFERENCIA DE LA VARIABLE RIESGO ANTE EVENTOS EXTREMOS (-1)

\begin{tabular}{lc}
\hline Categoría & Valor \\
\hline Riesgo alto & 0,33 \\
Riesgo medio-alto & 0,40 \\
Riesgo medio & 0,50 \\
Riesgo medio-bajo & 0,67 \\
Riesgo alto & 1,00 \\
\hline
\end{tabular}




\section{- $\quad$ Eje institucional}

Como menciona Rodríguez (2006), las instituciones como “...el mercado, la iglesia, la escuela, las autoridades, partidos politicos y sindicatos, organismos públicos y privados, organismos culturales, religiosos, económicos, políticos y artísticos...”, contribuyen al establecimiento de un sistema institucional arraigado en la cultura del lugar, que permite mediar en la afluencia de estrategias de comportamiento de los diferentes actores, al definir soluciones de cooperación en pro del desarrollo. De acuerdo con Vázquez Barquero (2002a), citado también por Rodríguez (2006), estas condiciones son determinantes para el éxito de un territorio. En esta línea, en este eje se agrupan una serie de indicadores que se aproximan mediante las variables que se detallan seguidamente.

\section{- Participación de la sociedad civil}

Un primer indicador que se identifica a la hora de evaluar la institucionalidad de un territorio es su participación civil y grado de asociatividad, (Rodríguez, 2006). Por ello, en la presente investigación este indicador se aproxima mediante las siguientes variables:

\section{Porcentaje de participación en elecciones presidenciales}

Corresponde al porcentaje de participación de los ciudadanos del cantón $i$ en las votaciones presidenciales. Los datos son generados por el Tribunal Supremo de Elecciones (TSE) y se encuentran disponibles para las elecciones presidenciales desde 1982 hasta el $2014^{16}$. En atención al objeto de la investigación, en esta primera etapa, únicamente se consideran los datos del año 2010. La variable podrá oscilar entre $0 \%$ en el caso de que los electores del cantón no hayan participado en las elecciones presidenciales y $100 \%$ si la totalidad de electores del cantón participaron en la elección.

\section{Porcentaje de participación en elecciones municipales}

Similar al indicador anterior, corresponde al porcentaje de participación de los ciudadanos del cantón i en las votaciones, solo que en este caso a nivel municipal. Los datos también son recopilados por el TSE y se encuentran disponibles para las elecciones municipales desde el 2006 hasta el $2010^{17}$. De acuerdo con el objeto de la investigación, en esta primera etapa únicamente se considera el año 2010.

Dicha variable puede variar entre $0 \%$, en el caso de que los electores del cantón no hayan participado en las elecciones municipales y $100 \%$ en el que la totalidad de los electores participan en la elección municipal.

\footnotetext{
16 En Costa Rica las elecciones presidenciales se realizan cada 4 años.

17 En Costa Rica las elecciones municipales se realizan cada 4 años, a excepción del año 2010 en que se realizó por un periodo de 5 años.
} 


\section{- Seguridad ciudadana}

Otro indicador institucional de gran importancia es la seguridad ciudadana, por cuanto se infiere que a mayor seguridad ciudadana menor es el costo de prevención del delito (Rodríguez, 2006).

\section{Índice de Seguridad Ciudadana Cantonal (ISCc)}

Este indicador se aproxima mediante el Índice de Seguridad Ciudadana Cantonal (ISCc) ${ }^{18}$. El índice considera tres componentes que son la tasa de violencia doméstica estandarizada, la tasa de homicidio doloso estandarizada $y$ la tasa de robos $y$ hurto estandarizada. Dicho indicador puede variar entre un valor mínimo de cero, lo que significa que el cantón presenta el nivel mínimo de seguridad ciudadana y un valor máximo de aquel que representa el nivel máximo de seguridad ciudadana.

\section{- Planificación y gestión institucional}

Otro de los indicadores que se destaca a la hora de evaluar la institucionalidad de un territorio es el desempeño de las instituciones que operan dentro del mismo. De particular interés resulta la evaluación de los gobiernos locales, que tal como señala Cabrero et ál. (2003), se constituyen en promotores del desarrollo de las localidades. Así, para la medición de este indicador, se consideran los valores del IGM en las variables planificación, gestión financiera, control interno y recursos humanos para cada cantón.

Los valores obtenidos en estas variables oscilan entre $0 \%$, ante el incumplimiento total de las metas programas por parte de las municipalidades; $y$ 100\%, para aquellos que cumplan plenamente con las metas programadas.

\section{- Instituciones financieras}

La presencia de instituciones requeridas para el desarrollo de las actividades económicas en un territorio es otra de las variables clave para la identificación de zonas con potencial de desarrollo productivo. Su medición se aproxima mediante la siguiente variable:

\section{Instituciones financieras por cada 1000 habitantes}

La presencia de instituciones financieras reduce los costos de transacción que las empresas deben asumir para llevar a cabo su actividad productiva. Por ello, se construye una variable que parte de los datos de bancos del Atlas Digital de Costa Rica 2008 del Tecnológico de Costa Rica (TEC) y la proyección de población del Centro Centroamericano de Población (CCP) para el año 2008, aplicando la distribución de población cantonal del Censo 2011del INEC.

$$
\text { Bancos por cada mil habitantes }{ }_{i}=\frac{\text { Bancos }_{i}}{\operatorname{Pob}_{i}} 1000
$$

18 El ISCc se incluye en el Atlas de Desarrollo Humano Cantonal Costa Rica 2011, elaborado de manera conjunta por el Programa de las Naciones Unidad para el Desarrollo (PNUD) y la Universidad de Costa Rica (UCR). Este fue calculado para los años 2005 y 2009 y, según consulta a los encargados del cálculo, se está trabajando en una nueva medición. Para la presente investigación se analizará el resultado general del índice en el año 2009. 
donde:

Bancos: $_{i}$ Cantidad de bancos del cantón ${ }_{i}$ $\mathrm{Pob}_{i}:$ Estimación de la población del cantón ${ }_{i}$

Dicha variable puede oscilar entre un valor mínimo de cero, lo que significa que el cantón no cuenta con presencia de bancos y un valor máximo que representa al cantón con mayor cantidad de bancos per cápita.

Acceso a centros de salud

La presencia de instituciones dedicadas a la atención de la salud es clave para el desarrollo social y productivo de un territorio, ya que permiten la atención de los cuidados de la salud de los pobladores de la localidad, lo cual incluye a los trabajadores y, por tanto, incide sobre sus capacidades y productividad.

En esa línea, para el caso de Costa Rica, este indicador se aproxima mediante la siguiente variable: EBAIS por cada 1000 habitantes. Para ello, se parte de las estadísticas actuariales de áreas y cantones de la Caja Costarricense del Seguro Social (CCSS) del año 2013 y del Censo 2011:

$$
\text { Ebais por cada mil habitantes } s_{i}=\frac{\text { Bancos }_{i}}{\operatorname{Pob}_{i}} * 1000
$$

donde:

Ebais. $_{\text {: }}$ Cantidad de Ebais del cantón $i$

Pob: Población del cantón $i$

Dicha variable puede fluctuar entre un valor mínimo de cero, lo que significa que el cantón no cuenta con presencia de Ebais y un valor máximo que representa al cantón con mayor cantidad de Ebais per cápita.

\section{- Instituciones educativas a nivel técnico}

Uno de los elementos clave para crear un entorno competitivo en un territorio es la educación (Millan, 1994). En ese sentido, la formación técnica juega un papel clave para contribuir con una oferta de recurso humano calificado y preparado para satisfacer las necesidades de las inversiones en actividades productivas que requieren este tipo de fuerza de trabajo. Por ello, se evalúan tres variables que buscan aproximar el grado de formación técnica con la que cuentan los cantones del país. Estas variables son las siguientes:

\section{Presencia de instituciones parauniversitarias}

Partiendo de los datos del Consejo Superior de Educación (CSE) de la República de Costa Rica, se construye una variable discreta con las siguientes categorías: 
CUADRO 4

VALORES DE REFERENCIA DE LA VARIABLE INSTITUCIONES PARAUNIVERSITARIAS

\begin{tabular}{lr}
\hline Categoría & Valor \\
\hline No presenta instituciones parauniversitarias & 0 \\
Presencia de instituciones parauniversitarias inactivas & 0,5 \\
Presencia de instituciones parauniversitarias activas & 1 \\
\hline
\end{tabular}

\section{Acceso a formación del INA}

Una de las instituciones llamada a cumplir un papel central en la formación del recurso humano que el país requiere para atender las demandas por fuerza de trabajo más calificada es el Instituto Nacional de Aprendizaje (INA). En consecuencia, una de las variables que se incorpora en la investigación es el acceso que la población de los distintos cantones tiene a los cursos ofrecidos por el INA en las diferentes áreas de especialización técnica.

$$
\text { Acceso a formación del INA } A_{i}=\frac{\text { Matricula }_{\mathrm{i}}}{\mathrm{Pob}_{i}} * 1000
$$

donde:

Matrícula: Cantidad de cursos matriculados por los habitantes del cantón $i$ Pob $_{i}$ : Población del cantón $i$

Dicha variable puede fluctuar entre un valor mínimo de cero, lo que significa que las personas del cantón no tuvieron acceso a matricular cursos del INA y un valor máximo que representa al cantón con la mayor matrícula per cápita de cursos del INA.

\section{- Colegios técnicos por cada 10000 habitantes}

Una última variable para medir el acceso a instituciones educativas a nivel técnico es la cantidad de colegios técnicos por cada 10000 habitantes $^{20}$ en cada cantón. Esta información se obtiene a partir de los datos del Atlas Digital de Costa Rica 2008 del Instituto Tecnológico de Costa Rica (TEC) y se plantea como sigue:

$$
\text { Colegios técnicos por cada } 10000 \text { habitantes }_{i}=\frac{\mathrm{Tec}_{i}}{\mathrm{Pob}_{i}} * 10000
$$

donde:

Tec $c_{i}$ Cantidad de colegios técnicos en el cantón $i$

$\mathrm{Pob}_{i}$ : Población del cantón $i$

Dicha variable puede oscilar entre un valor mínimo de cero, lo que significa que el cantón no cuenta con colegios técnicos y un valor máximo que representa al cantón con mayor presencia de colegios técnicos per cápita.

20 Los datos de población se obtienen a partir de la proyección de población del CCP para el año 2008, tras aplicar las proporciones según cantón del Censo 2011. 
Procedimiento metodológico para la construcción del índice de potencial productivo cantonal y análisis de conglomerados

Una vez que ya se cuenta con las variables a estudiar, se procede con el análisis y evaluación de estas. Para ello, se aplica un procedimiento metodológico que consta de dos etapas, a saber: la construcción de un índice de potencial productivo cantonal y la aplicación de un análisis de conglomerados.

\section{Etapa 1. Construcción del índice}

Paso 1. Se obtienen los valores de las variables que se incluyen en el índice. Primeramente, se realiza su cálculo o transformación, de manera que cumplan con el aporte esperado en cuanto a capacidad de discriminación y su relación con la calificación dentro del índice. Posteriormente, se transforman sus valores para poder ser incluidos en el índice. Finalmente, se estandarizan los valores para obtener resultados que faciliten su comparación e interpretación ${ }^{21}$.

Paso 2. Se asignan pesos a las variables y ejes del índice. Al no contar con un criterio científico definido $y$ validado previamente, se asigna el mismo peso a los tres ejes que componen el índice (especialización productiva, infraestructura e instituciones) ${ }^{22}$. De igual forma sucede con los variables, a las cuales se le asigna la misma ponderación dentro del eje, con excepción de las asociadas a los indicadores gestión ambiental, planificación y gestión e, instituciones educativas a nivel técnico; cuyas variables que los componen, se distribuyen en forma homogénea el mismo peso relativo que se asigna a las otras variables dentro del eje (ver cuadro 5).

21 Para la estandarización y transformación, se aplica una función exponencial a los valores obtenidos por cada uno de los cantones en los tres ejes que componen el cálculo del índice; posteriormente, se calcula el cociente de este valor con respecto al valor máximo cantonal obtenido en cada eje. Este método se selecciona al ser el que muestra una mayor capacidad de discriminación de los valores obtenidos por los cantones dentro de cada eje.

22 Estas ponderaciones podrían ser sujeto de ajuste, producto de los ejercicios de validación que realicen la segunda etapa del proyecto de investigación. 
CUADRO 5

PESOS ASIGNADOS A LAS VARIABLES DEL ÍNDICE

\begin{tabular}{|c|c|c|c|c|c|}
\hline $\begin{array}{l}\text { Variables del eje especializado } \\
\text { productiva }\end{array}$ & Peso & $\begin{array}{l}\text { Variables de eje } \\
\text { Infraestructura }\end{array}$ & Peso & Variables del eje Institucional & Peso \\
\hline $\begin{array}{l}\text { Generación de empleo total } \\
\text { (volumen) }\end{array}$ & 33,33 & $\begin{array}{l}\text { Tenencia y estado del plan } \\
\text { regulador }\end{array}$ & 11,11 & $\begin{array}{l}\text { Porcentaje de participación en } \\
\text { elecciones presidenciales }\end{array}$ & 14,29 \\
\hline $\begin{array}{l}\text { Porcentaje de empleo real con } \\
\text { secundaria completa o más } \\
\text { (calidad) }\end{array}$ & 33,33 & Gestión vial & 11,11 & $\begin{array}{l}\text { Porcentaje de participación en } \\
\text { elecciones municipales }\end{array}$ & 14,29 \\
\hline \multirow[t]{10}{*}{$\begin{array}{l}\text { Porcentaje de empleo } \\
\text { proveniente de otras localidades }\end{array}$} & 33,33 & $\begin{array}{l}\text { Distancia respecto al } \\
\text { puerto más cercano }(-1)^{*}\end{array}$ & 11,11 & $\begin{array}{l}\text { Índice de seguridad ciudadana } \\
\text { cantonal (ISCc) }\end{array}$ & 14,29 \\
\hline & & $\begin{array}{l}\text { Distancia respecto a } \\
\text { aeropuertos }(-1)\end{array}$ & 11,11 & Planificación & 3,57 \\
\hline & & $\begin{array}{l}\text { Distancia respecto a la } \\
\text { frontera mas cercana }(-1)\end{array}$ & 11,11 & Gestión financiera & 3,57 \\
\hline & & $\begin{array}{l}\text { Penetración de Banda } \\
\text { Ancha }\end{array}$ & 11,11 & Recursos humanos & 3,57 \\
\hline & & $\begin{array}{l}\text { Depósito y tratamiento de } \\
\text { residuos }\end{array}$ & 3,70 & Control interno & 3,57 \\
\hline & & Aseo de vias & 3,70 & $\begin{array}{l}\text { Instituciones financieras por } \\
\text { cada } 1000 \text { habitantes }\end{array}$ & 14,29 \\
\hline & & Recolección de residuos & 3,70 & Ebais por cada 1000 habitantes & 14,29 \\
\hline & & $\begin{array}{l}\text { Precio por metro } \\
\text { cuadrado según actividad } \\
(-1)\end{array}$ & 11,11 & $\begin{array}{l}\text { Presencia de instituciones } \\
\text { parauniversitarias }\end{array}$ & 4,76 \\
\hline & & $\begin{array}{l}\text { Riesgo ante eventos } \\
\text { extremos }(-1)\end{array}$ & 11,11 & Accseso a formación del INA & 4,76 \\
\hline & & & & $\begin{array}{l}\text { Colegios técnicos por cada } 10 \\
000 \text { habitantes }\end{array}$ & 4,76 \\
\hline
\end{tabular}

* El (-1) denota una relación inversa entre la variable y su aporte al eje, por ello, los valores originales se transforman para incluirse en el índice.

Nota. Dentro del índice se asigna el mismo peso a cada uno de los ejes, es decir, 33,33.

Paso 3. Se aplican las ponderaciones presentadas en el paso 2 a los valores que presentan los cantones en cada variable. Al aplicar los pesos respectivos a las variables, se obtiene un valor sub índice dentro de cada eje. Posteriormente, se aplica la ponderación de cada uno de los ejes a estos valores sub índice, obteniendo un valor asociado a cada eje. La suma de estos valores conforma el resultado del índice para cada cantón.

Tras la aplicación de esta primera etapa se obtendrá como resultado una clasificación cantonal en el índice y en cada uno de los ejes. Los valores obtenidos en el índice presentan una oscilación entre 0 como el menor potencial productivo posible y 1 como el máximo.

Paso 4. Análisis de estadísticos descriptivos básicos. Se realiza un análisis de estadísticos descriptivos básicos en el índice y dentro de cada uno de los ejes que lo componen (subíndices). Los estadísticos seleccionados son: media (X), desviación estándar (), rango y correlación lineal. 
Etapa 2. Análisis de conglomerados

Paso 1. Se aplica el método de análisis de conglomerados para agrupar los cantones según el valor que obtengan en el índice. Primeramente se selecciona el algoritmo de formación de conglomerados, en nuestro caso es el de partición, en el que se definen k=5 conglomerados; es decir, los cantones se clasifican en 5 grupos de acuerdo con el puntaje que obtengan dentro del índice, como sigue:

CUADRO 6

CONGLOMERADOS EN LOS QUE SE CLASIFICAN LOS CANTONES SEGÚN EL VALOR OBTENIDO EN EL ÍNDICE

\begin{tabular}{cc}
\hline Conglomerado & Valor \\
\hline Conglomerado 1 & Potencial alto \\
Conglomerado 2 & Potencial medio-alto \\
Conglomerado 3 & Potencial medio \\
Conglomerado 4 & Potencial medio-bajo \\
Conglomerado 5 & Potencial bajo \\
\hline
\end{tabular}

Como resultado de este paso se obtendrá una clasificación cantonal en 5 conglomerados, de acuerdo con su potencial de desarrollo productivo, lo que se podría interpretar como su capacidad para constituirse en un "núcleo" para el establecimiento de una ZEED.

Paso 2. Análisis de estadísticos descriptivos básicos dentro de los conglomerados. Se realiza un análisis de estadísticos descriptivos básicos de cada uno de los conglomerados. Los estadísticos seleccionados son: media (X), desviación estándar ( ), rango y correlación lineal.

Paso 3. Análisis de conglomerados dentro de los ejes: Se aplican los pasos 1 y 2 para cada uno de los ejes y posteriormente se realiza un análisis de estadísticos descriptivos.

Como resultado se obtendrá la clasificación de los cantones en conglomerados ${ }^{23}$, de acuerdo con su potencial dentro de cada uno de los ejes.

\section{RESULTADOS}

En esta sección se muestran los resultados de la aplicación metodológica seleccionada para cumplir con el objeto del presente estudio. Este consta de dos etapas; la primera, en la que se construye un índice con las variables seleccionadas; $y$ la segunda, en la que se realiza un análisis de conglomerados, con el fin de agrupar los cantones según su potencial de desarrollo productivo, tanto a nivel del índice como dentro de cada uno de los ejes que lo componen.

Análisis del índice de potencial productivo

Tras la construcción del índice cantonal de potencial productivo, se obtiene un valor para cada uno de los cantones en el índice y el aporte realizado por los ejes que lo componen. El resulta$23 \quad$ Las categorías son las mismas que las descritas para el caso de los conglomerados a nivel del índice. 
do se resume en el gráfico 1, que muestra la posición relativa de los 81 cantones en orden descendente, así como el aporte que realiza cada uno de los ejes a la calificación obtenida por el cantón. Esto posibilita una comparación de los cantones en el índice, así como de sus ejes y su contribución relativa, aproximando un perfil cantonal de potencial productivo.

En esa línea, se observa que Belén es el cantón que presenta un mayor potencial productivo relativo, con un valor de 0,70 , siendo el eje institucional el que realiza una mayor contribución en la calificación, seguido por los ejes infraestructura y especialización productiva, respectivamente. Por el contrario, Siquirres es el cantón con el menor potencial de desarrollo productivo relativo $(0,15)$, siendo el eje infraestructura el que más aporta en su calificación, le siguen los ejes institucional y especialización productiva, en ese orden.

Así mismo, se observa que el promedio de calificación en el índice es de 0,35, destacándose que en términos generales el eje infraestructura es el que realiza un mayor aporte en la calificación, seguido por el institucional y finalmente el de especialización productiva. Además, el índice presenta una variabilidad alrededor de la media de 0,12 puntos. Referente al nivel de variación dentro de los ejes, este es relativamente menor al del índice, oscila entre 0,05 y 0,07 .

\section{GRÁFICO 1}

COMPOSICIÓN DEL ÍNDICE CANTONAL PARA LA IDENTIFICACIÓN Y DEFINICIÓN DE REGIONES CON ALTO POTENCIAL DE DESARROLLO PRODUCTIVO PARA EL ESTABLECIMIENTO DE ZEED

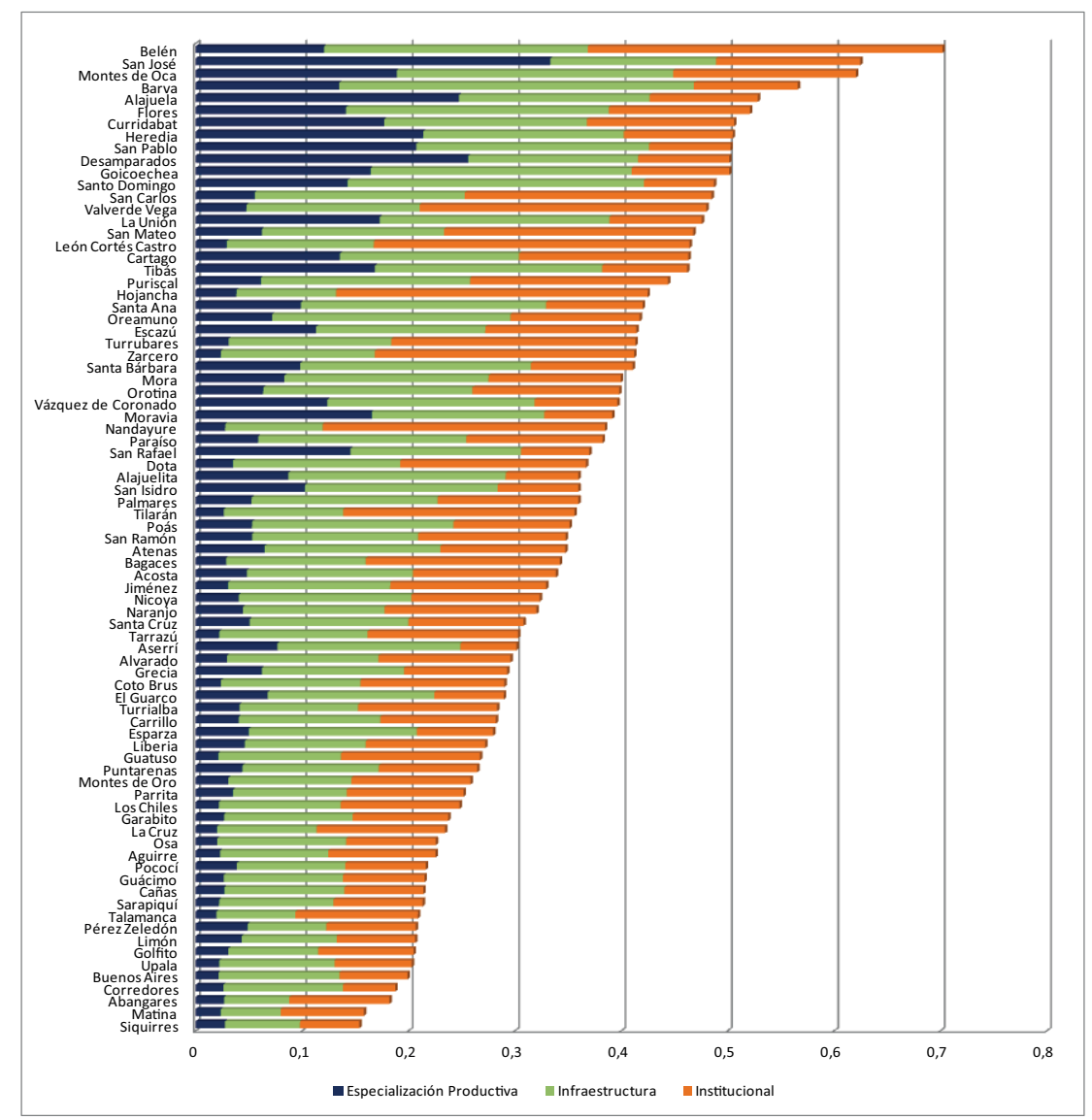

Fuente: Elaboración propia. 
Otro análisis que resulta de interés es el del grado de correlación existente entre el valor del índice y el aporte de los diferentes ejes, ya que nos permite conocer su grado de asociación. Se destaca que el eje infraestructura es el que presenta una mayor relación con el índice $(0,80)$, seguido por eje especialización productiva $(0,74)$, ambas las podríamos categorizar como fuertes.

Por su parte, el eje institucional es el que muestra una menor relación lineal con el valor del índice $(0,47)$; no obstante, este valor se podría interpretar como un nivel de correlación que se aproxima a fuerte ${ }^{24}$. En general, se resalta que el aporte de los tres ejes presenta un importante grado de asociación con la calificación que los cantones obtienen en el índice, es decir, en términos generales, si un cantón obtiene una mayor calificación en cada uno de los ejes, es de esperar que obtenga una mayor calificación en el índice.

En cuanto a la correlación entre los diferentes ejes, también resulta de interés ver en qué medida los aportes que realizan estos, están asociados entre sí; lo que podría significar que los cantones presentan fortaleza relativa en más de uno de los ejes. A continuación, se resume el análisis de correlación respectivo.

\section{FIGURA 1}

ANÁLISIS DE CORRELACIÓN ENTRE LOS EJES QUE COMPONEN EL ÍNDICE

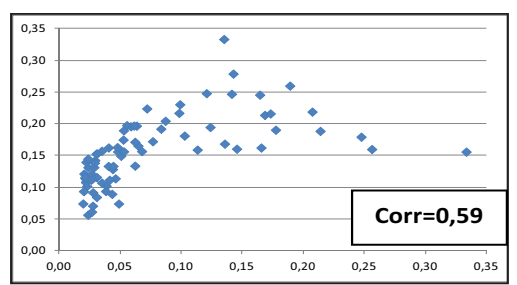

Correlación especialización productiva-infraestructura: Estos dos ejes presentan una correlación lineal fuerte; es decir, en general se podría afirmar que tener un potencial productivo a nivel de especialización productiva está relacionado con tener potencial a nivel de infraestructura.

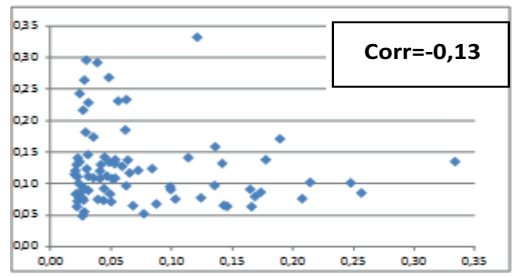

Correlación especialización productiva-institucional: Se aprecia una asociación negativa (cercana a cero), lo que representa poca asociación e inversa de las calificaciones que se presentan en ambos ejes.

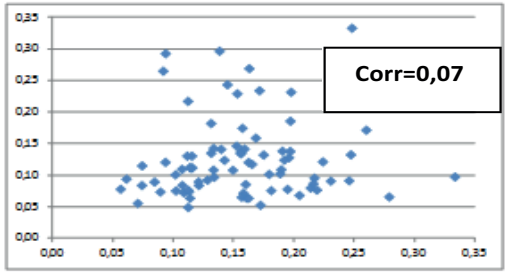

Correlación infraestructura-institucional: Se observa que ésta es cercana de cero, lo que significa que las calificaciones obtenidas en estos ejes prácticamente no están relacionadas.

Fuente: Elaboración propia.

24 Para efectos del presente análisis y en concordancia con los valores usualmente aceptados, un valor de correlación lineal a partir de 0,50 se considera fuerte. 
Análisis de conglomerados

\section{Conglomerados a nivel del índice}

Una vez que se cuenta con los resultados del índice tanto a nivel general como dentro de cada eje, se aplica un análisis de conglomerados que clasifica los cantones en 5 grupos de acuerdo con su potencial de desarrollo productivo para el establecimiento de ZEED, a saber: alto, medio-alto, medio, medio-bajo y bajo. La figura 1 muestra los resultados de la aplicación a nivel del índice en su conjunto:

FIGURA 2

CONGLOMERADOS A NIVEL DEL ÍNDICE

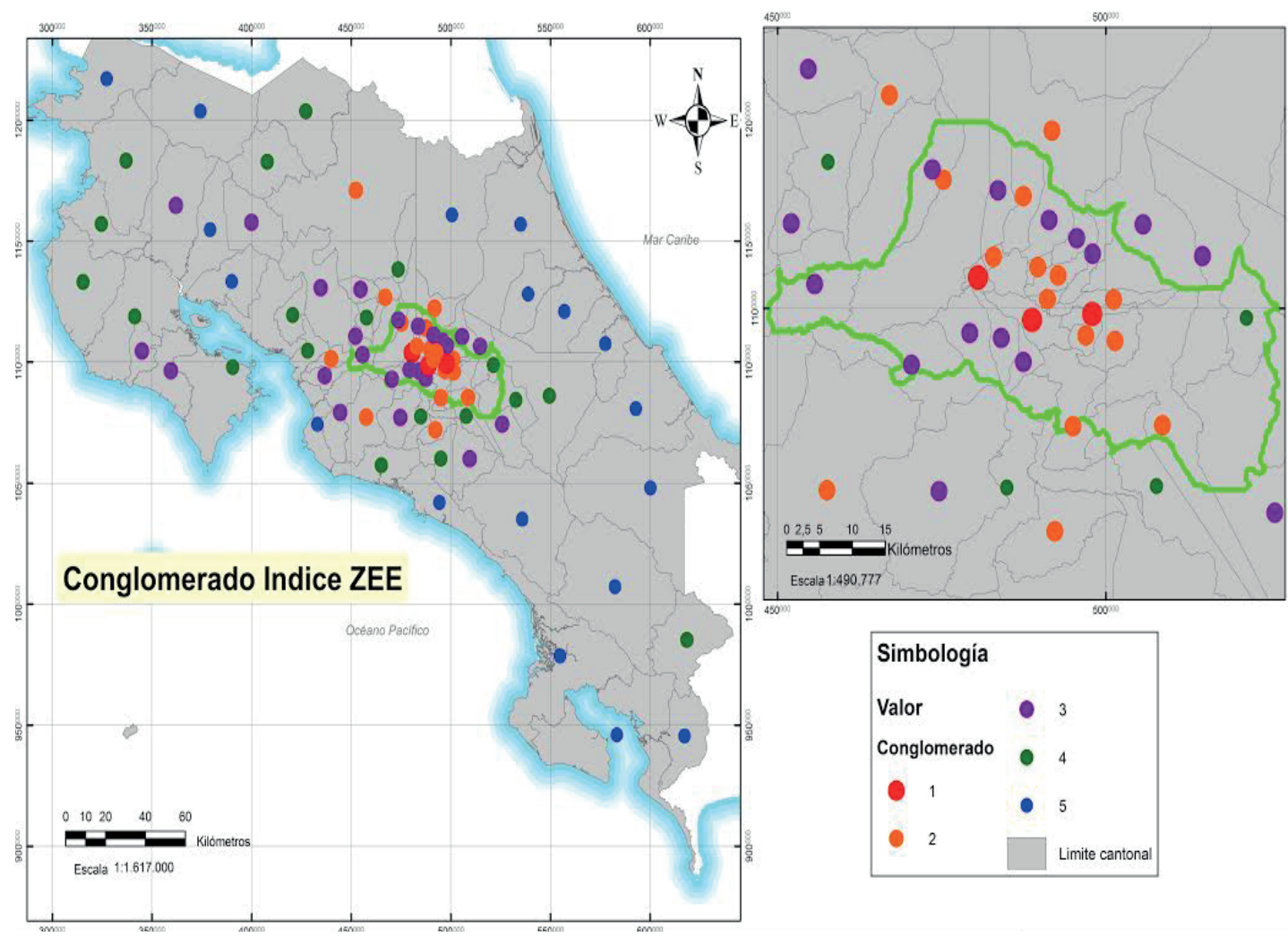

Fuente: Elaboración propia.

Del mapa, se destaca que los cantones con un nivel de potencial productivo alto (Belén, Montes de Oca y San José) se concentran en la GAM. Estos cantones presentan un valor promedio en el índice de 0,65 , el cual es muy superior a la media cantonal $(0,35)$ y muy cercano al límite superior de 0,70. Resulta importante analizar cuál es, en términos generales, el aporte que realiza cada uno de los ejes al resultado del índice que obtienen los cantones que componen el conglomerado (gráfico 2). 
GRÁFICO 2

COMPOSICIÓN DEL ÍNDICE EN CONGLOMERADO 1: POTENCIAL ALTO

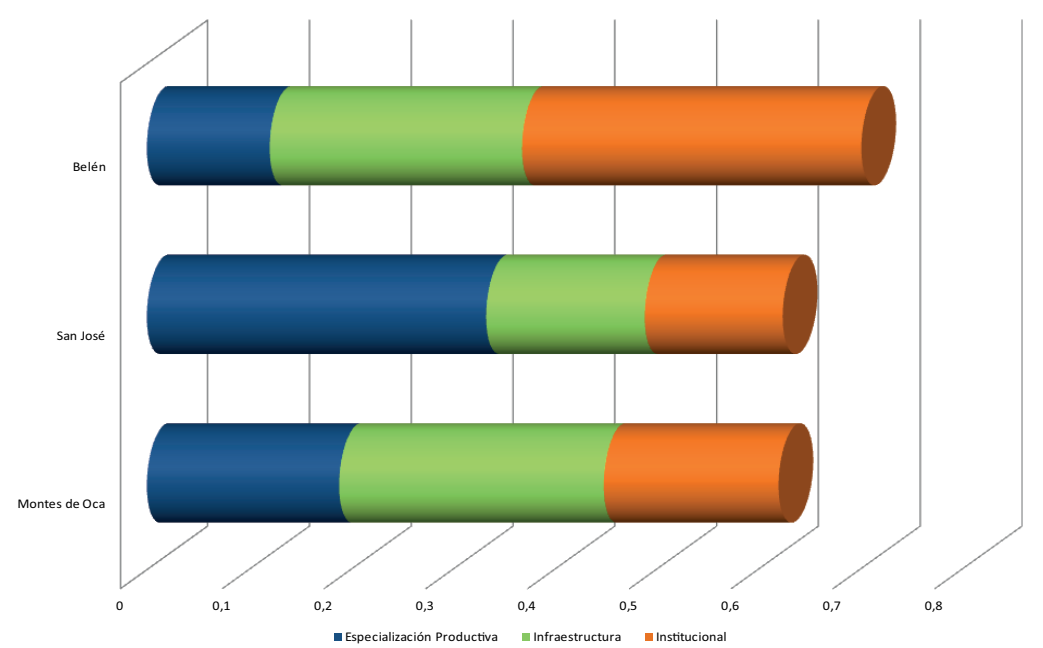

Fuente: Elaboración propia.

Se destaca cómo los cantones incluidos en este conglomerado cuentan con fortaleza relativa en los tres ejes, sin presentar dominancia de uno sobre los otros, ya que los cantones que conforman el conglomerado difieren en cuanto al eje que más aporta en el resultado del índice.

Respecto a los 17 cantones que conforman el conglomerado con potencial medio-alto, hay que destacar que solo cinco de ellos se encuentran fuera de la GAM (Puriscal, León Cortés Castro, San Mateo, Valverde Vega y San Carlos). Estos se ubican tanto hacia el Este como el Oeste del país; de tal forma que se podrían convertir en puentes que articulen el desarrollo productivo de la GAM con regiones periféricas con alto potencial de desarrollo productivo (gráfico 3 ).

\section{GRÁFICO 3}

COMPOSICIÓN DEL ÍNDICE EN EL CONGLOMERADO 2: POTENCIAL MEDIO-ALTO

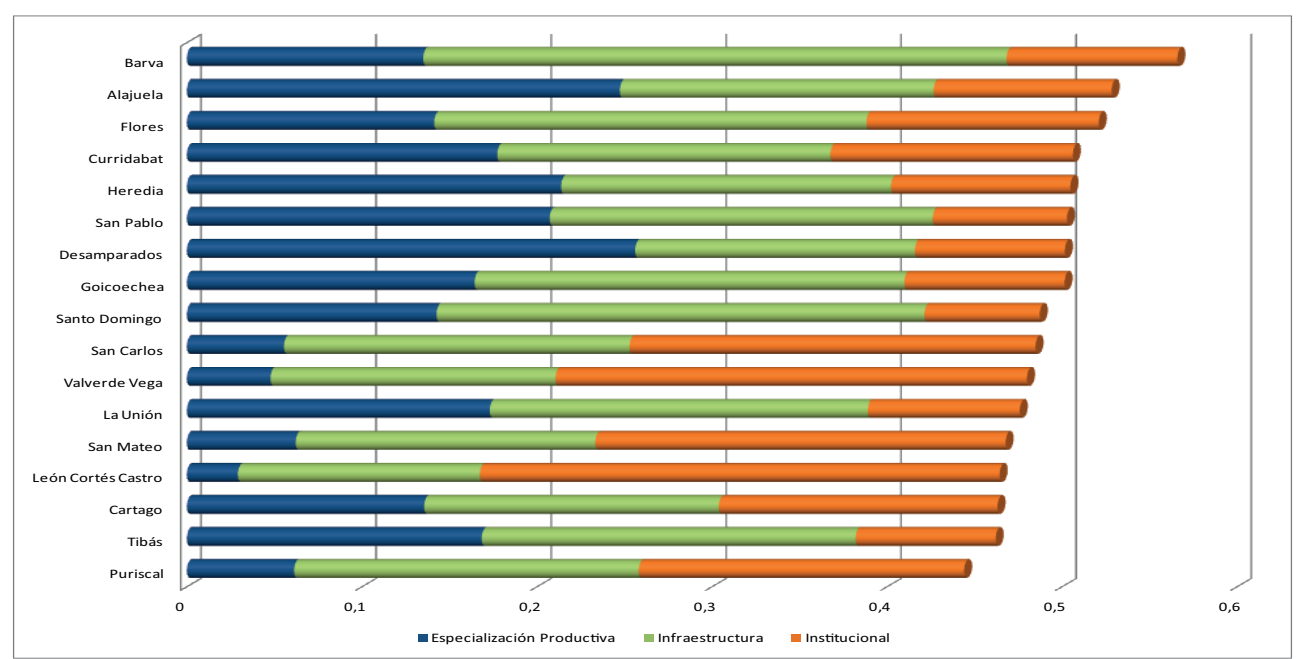

Fuente: Elaboración propia. 
Estos cantones presentan un valor promedio de 0,49, el cual es significativamente superior a la media del índice (0,35). Además, en general, el eje infraestructura es el que hace un mayor aporte a la calificación dentro del índice. La contribución de los otros dos ejes varía considerablemente según el cantón analizado. Por ejemplo, en el caso de León Cortés Castro el aporte del eje especialización productiva es relativamente bajo mientras que el institucional alto. Lo contrario sucede con Paraíso.

El tercer conglomerado es el de potencial medio, en el cual se destaca que cerca de la mitad de los 24 cantones que lo componen se encuentra fuera de la GAM, siendo importante resaltar que el valor promedio del índice en este grupo es de 0,38; es decir, ligeramente superior a la media cantonal. Referente a los cantones fuera de la GAM, estos se ubican en sus periferias Este y Oeste, con una mayor concentración hacia el suroeste, formándose un corredor hacia el Pacífico que pasa por varios cantones de la provincia de Alajuela y se extiende hasta territorios pertenecientes a las provincias de Puntarenas $y$ Guanacaste.

Como se destaca en el siguiente gráfico, los ejes infraestructura e institucional son los dos que realizan una mayor contribución a la calificación en el índice obtenida por los cantones que componen el conglomerado, mientras que la especialización productiva presenta un menor aporte relativo.

\section{GRÁFICO 4}

\section{COMPOSICIÓN DEL ÍNDICE EN EL CONGLOMERADO 3: POTENCIAL MEDIO}

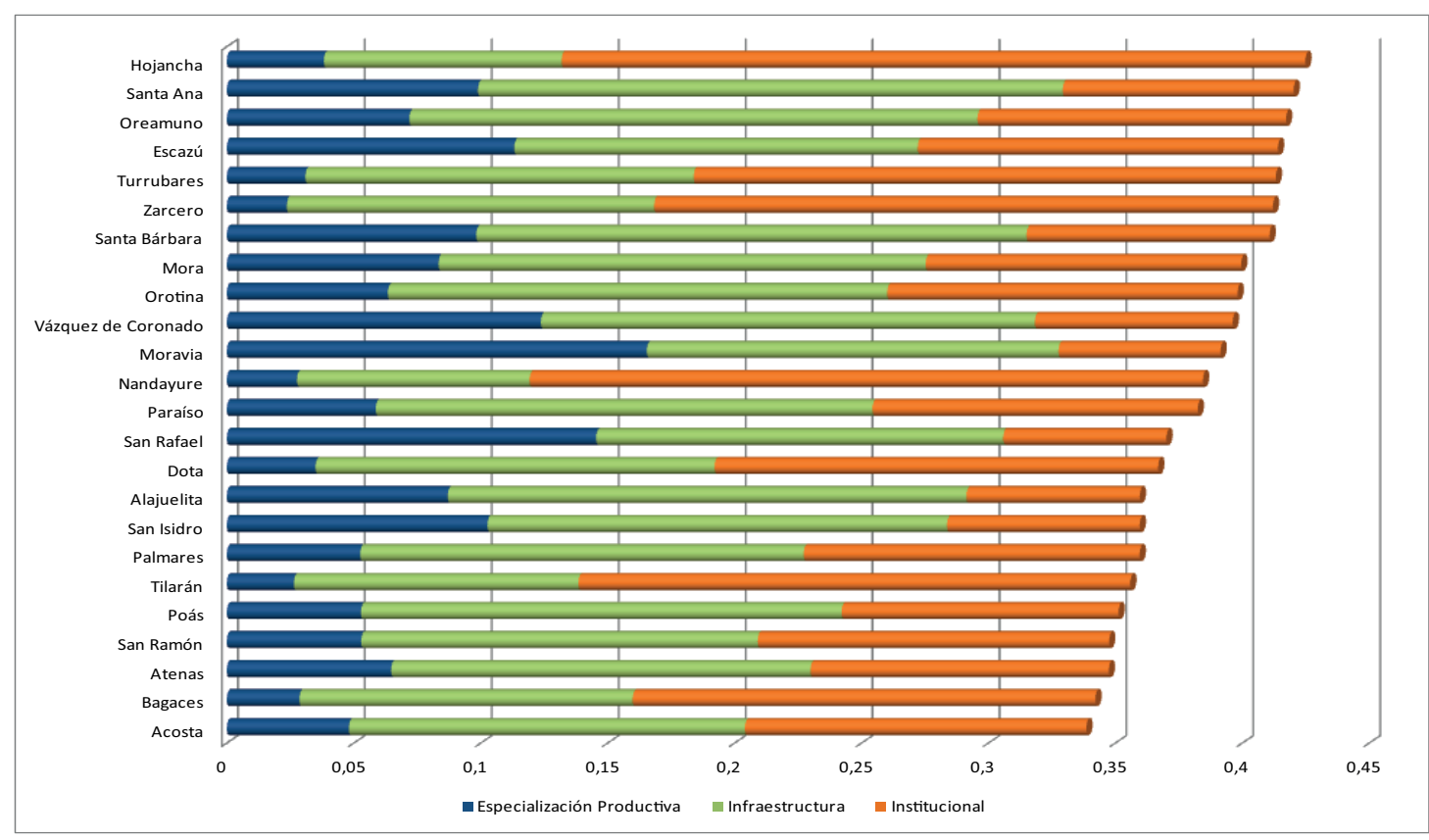

Fuente: Elaboración propia.

El cuarto grupo que se conforma en torno a los valores del índice es el de potencial mediobajo, que se encuentra conformado por 19 cantones de los cuáles únicamente 3 (dos de ellos parcialmente) forman parte de la GAM. El valor promedio del índice en este grupo es de 0,29, el cual es ligeramente inferior a la media del índice $(0,35)$. Seguidamente la composición de la calificación según eje. 


\section{GRÁFICO 5 \\ COMPOSICIÓN DEL ÍNDICE EN EL CONGLOMERADO 4: POTENCIAL MEDIO-BAJO}

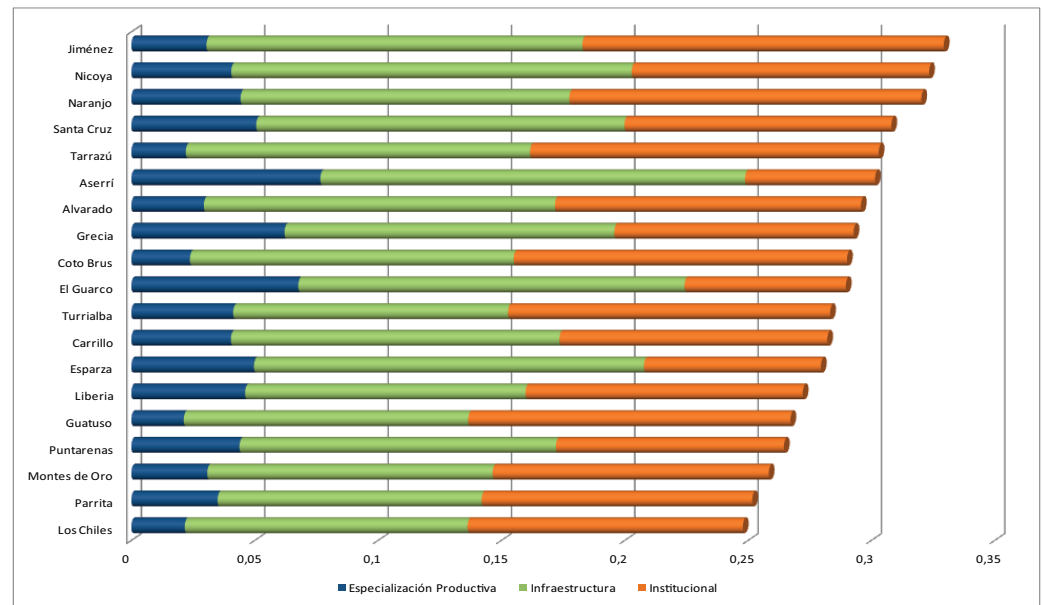

Fuente: Elaboración propia.

Se puede apreciar que el eje que realiza un mayor aporte relativo en la calificación del índice es el de infraestructura, seguido por el institucional y finalmente con una contribución significativamente menor el eje especialización productiva.

Por último, se conforma un conglomerado que agrupa los 18 cantones con la menor calificación relativa dentro del índice, cuya calificación promedio es de 0,21 , valor muy inferior al promedio nacional. Respecto de la posición geográfica de estos cantones, ninguno de ellos forma parte de la Gran Área Metropolitana y se encuentran a distancias relativamente largas de ella, que se constituye como el epicentro de la actividad económica del país. Referente al aporte de los ejes a la calificación del índice, se resalta que el eje infraestructura es el que realiza una mayor contribución, seguido por los ejes institucional y especialización productiva respectivamente, tal y como se aprecia en el siguiente gráfico 6.

GRÁFICO 6

COMPOSICIÓN DEL ÍNDICE EN EL CONGLOMERADO 5: POTENCIAL BAJO

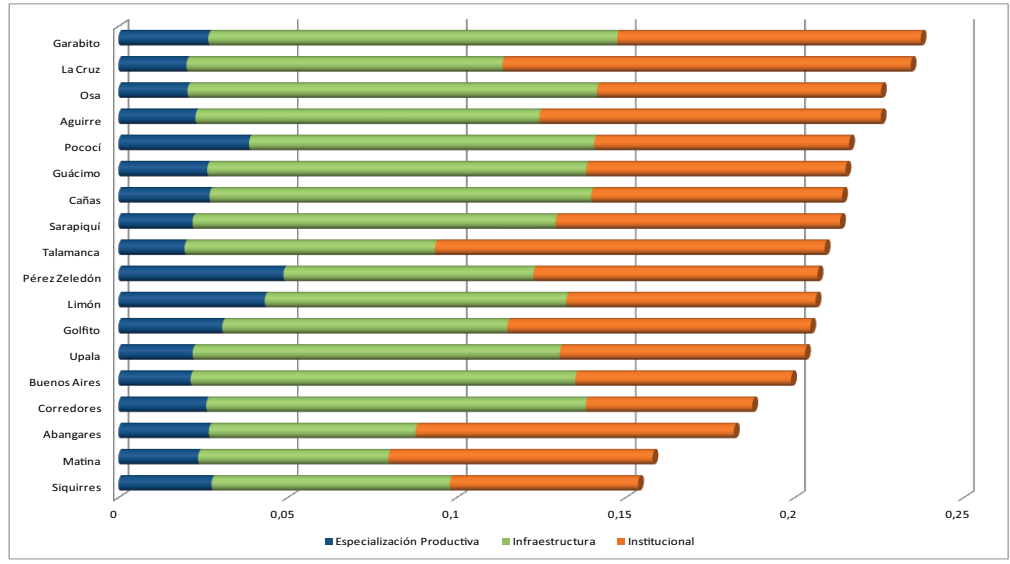

Fuente: Elaboración propia. 
Otro de los resultados que es de gran interés es la formación de conglomerados dentro de cada uno de los ejes que componen el índice. De esta manera, se agrupan los cantones según su fortaleza relativa en el componente especialización productiva, infraestructura e institucional.

\section{Conglomerados en el eje especialización productiva}

En la figura 3 se muestran los resultados de la aplicación de la técnica en el eje especialización productiva.

\section{FIGURA 3}

\section{CONGLOMERADOS EN EL EJE ESPECIALIZACIÓN PRODUCTIVA}

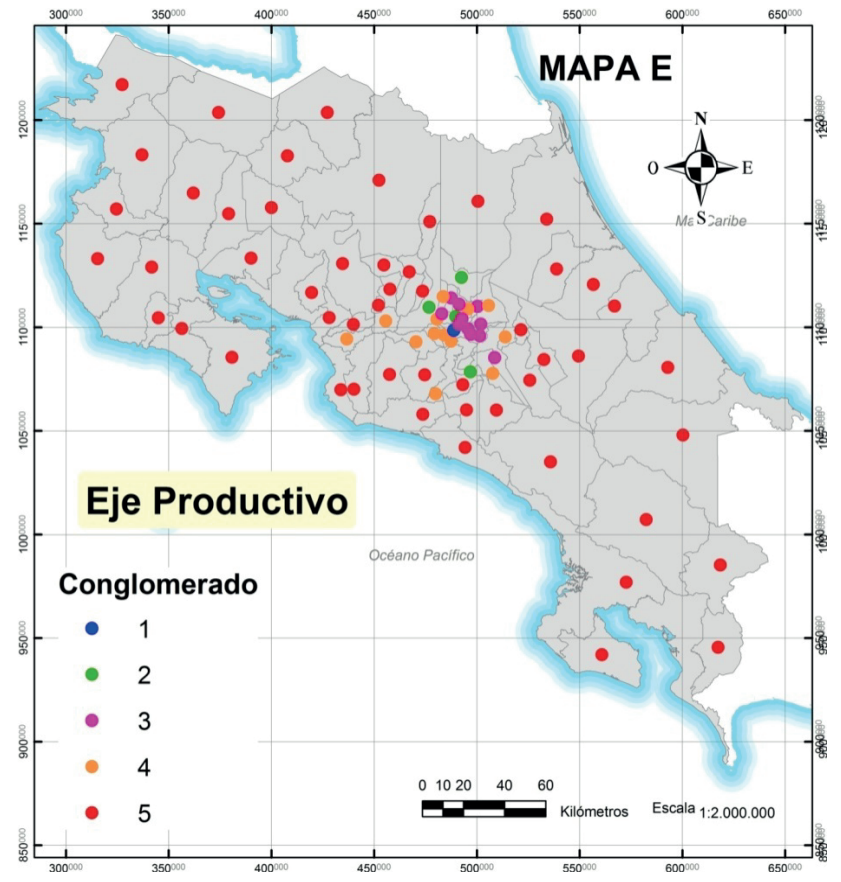

Fuente: Elaboración propia.

Como se observa en el mapa, el único cantón que conforma el conglomerado con potencial alto en el eje es San José, lo cual se explica por la alta concentración de mano de obra que vive en este cantón y la que se desplaza de cantones vecinos.

Un segundo grupo en este eje es compuesto por 4 cantones, de los cuales tres de ellos son cabecera de 3 de las 4 provincias que componen la GAM. El aporte promedio que realiza este eje al índice en estos cantones es de 0,23 puntos con respecto a un valor máximo de 0,33 , lo que presenta un potencial medio-alto.

El grupo de cantones que conforman el tercer conglomerado, potencial medio, realizan un aporte promedio al índice de 0,16 , es decir, de casi $50 \%$ del aporte máximo posible. Esto se podría interpretar como un potencial medio en especialización productiva. Se destaca que los 11 integrantes del conglomerado forman parte de la GAM. 
Respecto al cuarto conglomerado, en este se ubican 13 cantones, de los cuales 12 forman parte de la GAM, con excepción de Orotina, que es un cantón de la provincia de Alajuela y colindante con la provincia de Puntarenas, el cual podría constituirse como un articulador de desarrollo productivo de la GAM con territorios vecinos o conectados por infraestructura vial, como es el corredor San José-Puerto Caldera. El aporte medio que realizan los cantones del grupo en la calificación del índice es de 0,09 , lo que representa un $27 \%$ de la contribución máxima que podrían realizar a la calificación del índice.

Finalmente, se identifica un quinto conglomerado que agrupa 52 de los 81 cantones del territorio nacional, cuyo aporte promedio a la calificación del índice es de 0,04; apenas el 12\% del aporte máximo que podría realizar. De estos, solo 2 se ubican en la GAM, los restantes se distribuyen a lo largo del territorio nacional.

\section{Conglomerados en el eje infraestructura}

En el caso del eje de infraestructura, nos encontramos con los siguientes resultados.

\section{FIGURA 4}

\section{CONGLOMERADOS EN EL EJE INFRAESTRUCTURA}

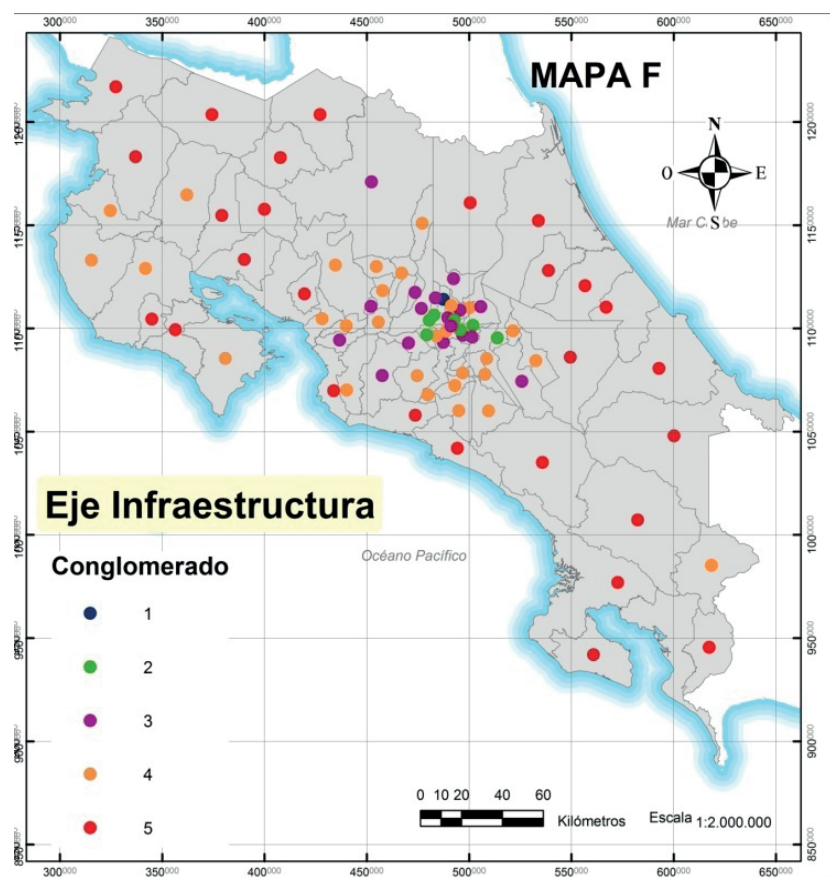

Fuente: Elaboración propia.

Como se aprecia en el mapa, el único cantón que conforma un conglomerado con potencial alto en el eje infraestructura es Barva, el cual pertenece a la GAM y más específicamente a la provincia de Heredia. 
Un segundo conglomerado con potencial medio-alto está conformado por 7 cantones de la GAM. Estos realizan un aporte promedio al resultado del índice de 0,25, lo que representa prácticamente un $70 \%$ del aporte máximo que podrían realizar.

El conglomerado con potencial medio agrupa 17 cantones, los cuales muestran un aporte promedio de 0, 20 (casi un 60\% del aporte máximo posible). Por otra parte, se destaca que solo 5 de estos cantones se localizan fuera de la GAM, tanto al Este como al Oeste (Puriscal, Vásquez de Coronado, Palmares, Orotina y San Carlos). Estos cantones podrían constituirse en importantes núcleos para articular el desarrollo productivo de la GAM con otras regiones y generar mayores niveles de convergencia y expansión del desarrollo productivo.

El grupo número 4 (potencial medio-bajo) está compuesto por 29 cantones, estando más del 65\% fuera de la GAM. La contribución media a la calificación del índice por parte de este eje dentro del índice en este grupo es 0,15 ; poco menos del 50\% del aporte que podrían realizar.

El quinto y último conglomerado dentro del eje, potencial bajo, está conformado por 27 cantones ubicados fuera de la GAM. Estos realizan un aporte promedio al índice de 0,10, es decir, el $30 \%$ del máximo aporte posible. Como se puede apreciar en el mapa, los cantones de este grupo se ubican en la periferia territorial del país, concentrándose a lo largo de sus costas y fronteras.

\section{Conglomerados en el eje institucional}

Referente al eje institucional, también se realiza un análisis de conglomerados que clasifica los cantones en 5 grupos (figura 5).

FIGURA 5

CONGLOMERADOS EN EL EJE INSTITUCIONAL

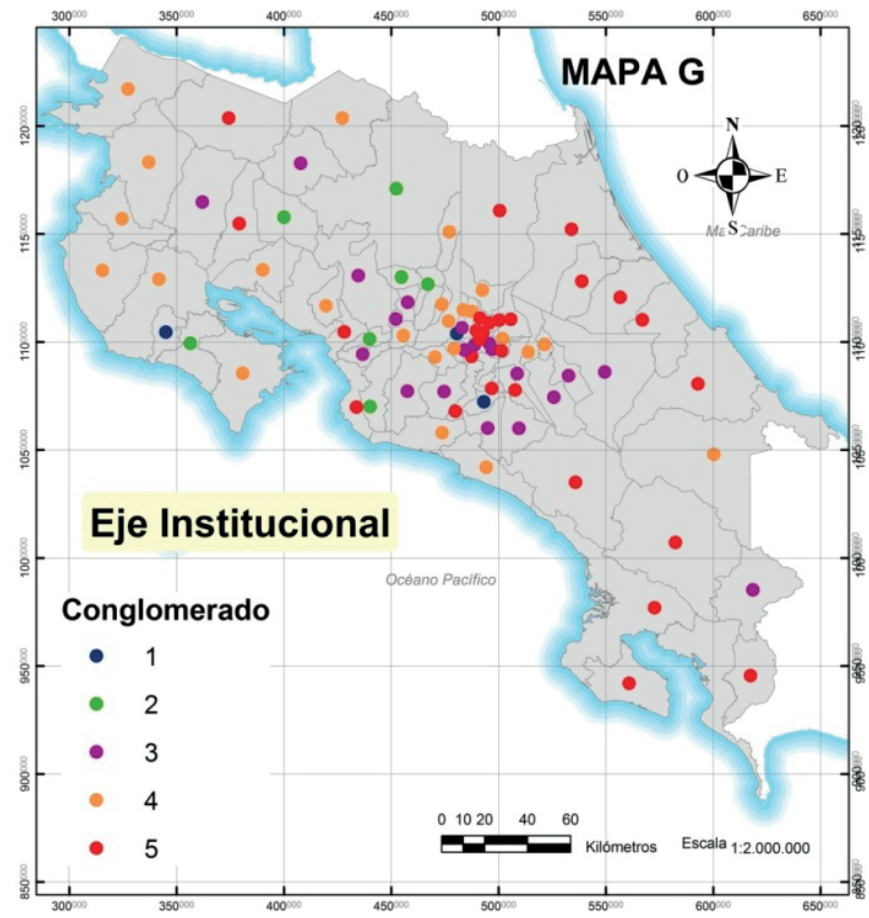

Fuente: Elaboración propia. 
Del mapa se aprecia que el conglomerado con potencial alto lo componen tres cantones (Belén, León Cortés y Hojancha). Se resalta el caso de Belén que ocupa la calificación máxima en este eje así como en el índice en su conjunto. También llama la atención el resultado de Hojancha, que es el único cantón fuera de la GAM que se ubica en un conglomerado de alto potencial. Este está situado en la costa pacífica del país, en la provincia de Guanacaste. En promedio, los cantones que conforman este conglomerado aportan 0,31 a la calificación del índice, lo que representa prácticamente el valor máximo posible de 0,33 .

El segundo conglomerado con potencial medio-alto consta de 7 cantones, los cuales presentan un aporte promedio a la calificación del índice de 0,24 (73\% del máximo que podrían aportar). Se destaca que la totalidad de los cantones que componen este grupo se ubican fuera de la GAM (hacia el Suroeste) y mayoritariamente pertenecen a la provincia de Alajuela. Estos cantones podrían constituirse en un núcleo de desarrollo institucional para las regiones aledañas.

En cuanto al grupo de 20 cantones con potencial medio (conglomerado 3), la contribución promedio que estos realizan al resultado del índice es 0,15; es decir, aproximadamente el $44 \%$ del valor máximo posible. Se resalta que el $60 \%$ de los cantones pertenecientes a este grupo están fuera de la GAM, los cuales podrían representar una alternativa para el fortalecimiento institucional de las regiones a las que pertenecen. Estos cantones muestran un cierto grado de concentración hacia el Suroeste del país y podrían cumplir un papel clave como articuladores de desarrollo en sus áreas de influencia.

Un cuarto conglomerado con potencial medio-bajo es conformado por 24 cantones, situados tanto dentro como fuera de la GAM. La contribución media que estos realizan a la calificación promedio del índice es de 0,11, aproximadamente el 33\% de la posibilidad máxima de aporte.

Finalmente, en un quinto conglomerado con potencial bajo, cuyo aporte a la calificación del índice es de tan solo 0,07 (el 22\% del aporte máximo posible). El conglomerado es conformado tanto por cantones pertenecientes al GAM como al resto del país. También resulta importante resaltar la ausencia de cantones ubicados en la provincia de Alajuela en este grupo.

\section{CONCLUSIONES}

Partiendo de los indicadores propuestos por la literatura y de acuerdo con la disponibilidad de datos para el caso costarricense, se seleccionó un conjunto de variables que miden una serie de aspectos que definen el potencial productivo de los cantones. Dichas variables se agrupan en tres grandes ejes: especialización productiva, infraestructura e institucional. Para el análisis y evaluación de las variables, se construyó un índice de potencial productivo cantonal y se aplicó el análisis de conglomerados.

Con la construcción del índice cantonal de potencial productivo, se aproxima un perfil de los 81 cantones que conforman territorialmente el país, sobre la base de sus características endógenas en cuanto a especialización productiva, infraestructura e instituciones.

La calificación promedio en el índice es de 0,35, con una variabilidad promedio de 0,12 y una concentración ligeramente superior hacia la cola inferior de la distribución, lo que implica que la mayoría de los cantones presentan calificaciones inferiores a la media cantonal, es decir, un potencial productivo relativamente bajo. Referente a los ejes, el de especialización productiva es el que presenta un aporte relativo menor y una mayor variabilidad entre cantones; los ejes infraestructura e institucional realizan aportes relativamente superiores en la calificación del índice y presentan una menor variabilidad entre los cantones.

Los cantones que ocupan las diez primeras posiciones dentro del índice se ubican en la GAM, siendo Belén, San José y Montes de Oca los que ocupan las tres primeras posiciones. Por otra parte, los cantones que ocupan las diez últimas posiciones se encuentran relativamente lejos de la GAM, los tres cantones con menor potencial productivo relativo son Siquirres, Matina y Abangares. 
En términos generales, el eje infraestructura es el que tiene un peso relativo mayor en la calificación del índice, seguido por el eje institucional y, finalmente, el eje de especialización productiva. Esto implica que, en términos generales, el resultado del eje infraestructura es el que tiene una mayor importancia relativa en la posición de los cantones en el índice de potencial productivo.

Así mismo, se presenta una correlación lineal relativamente fuerte entre el valor del índice y el aporte que realiza cada uno de los ejes que lo componen, es decir, es de esperar que entre mayor sea la calificación que presente un cantón en cada uno de los ejes, mayor es la calificación que este obtendrá en el índice.

Referente a la correlación entre las calificaciones dentro de los ejes, solo el aporte en especialización productiva e infraestructura se encuentran altamente correlacionados, lo que implica que en general si un cantón presenta un mayor potencial productivo en cuanto a infraestructura, también lo presenta en cuanto a especialización productiva, mientras que la asociación entre las otras calificaciones es prácticamente nula; es decir, en términos generales que un cantón presente fortaleza relativa en el eje institucional no implica que suceda lo mismo con los ejes infraestructura y especialización productiva.

A través del cálculo del índice para Costa Rica, se pudo constatar que es posible identificar zonas con potencial de desarrollo que se encuentran en la categoría media-alta y que no pertenecen a la GAM. Estas zonas tienen ventajas geográficas que se pueden explotar para localizar, desarrollar, y expandir nuevas industrias, ya que cuentan con las instituciones, la mano de obra y la infraestructura para la promoción y la atracción de nuevas inversiones.

Estas nuevas áreas se deben desarrollar según sus ventajas comparativas, dotación de recursos, tanto naturales como humanos, para la promoción de nuevos núcleos productivos. Es de ahí la importancia de poder definir e identificar las posibles nuevas áreas a desarrollar bajo el esquema de Zona Económica Especial de Desarrollo.

De la construcción y el cálculo del índice de ZEED también se concluye que hay áreas que pueden desarrollarse más, enfocándose en el fortalecimiento del eje donde tienen menor calificación. A partir de ahí cada cantón -según su calificación en cada eje- puede diseñar la política de desarrollo endógeno que responda a sus propias características, a la vez que mejore las condiciones de competitividad territorial necesarias para la atracción de inversiones y le imprima mayor dinamismo a las economías locales.

Las zonas con condiciones propicias para redefinir su política de desarrollo son aquellas que se encuentran dentro de la clasificación media, media-baja y baja. Claro está que el potencial de desarrollo que tienen esas zonas requiere además de políticas de inversión y de servicios públicos orientados con criterio territorial, para que en el mediano y largo plazo puedan constituir nuevos polos de acumulación y aglomeración económica del país.

Al aplicar el análisis de conglomerados a nivel del índice global, los cantones con mayor potencial de desarrollo productivo se ubican dentro de la GAM. Particularmente, se destaca que los cantones con potencial alto cuentan con fortaleza relativa en los tres ejes y no se observa dominancia de uno sobre los otros, ya que los cantones que conforman el conglomerado difieren en cuanto al eje que más aporta en el resultado del índice.

Se identifican cantones con alto potencial productivo tanto en la periferia Este como Oeste de la GAM. Además, se da una mayor concentración en el Suroeste del país, formándose un corredor hacia el Pacífico Central (Corredor San José-Puerto Caldera) que pasa por varios cantones de la provincia de Alajuela y se extiende hasta territorios pertenecientes a las provincias de Puntarenas y Guanacaste.

En términos generales, los conglomerados con un menor potencial productivo relativo están constituidos por cantones que se ubican en las periferias marítimas y terrestres del país, así como relativamente lejos de la GAM, zona que se constituye como el epicentro de la actividad productiva del país. 
Referente a los conglomerados a nivel del eje de especialización productiva, tres de los cuatro cantones que ocupan las primeras posiciones son cabecera de Áreas Metropolitanas del país (San José, Alajuela y Heredia). Otros cantones que destacan por su alto potencial en este eje son Desamparados y San Pablo, lo que refuerza el argumento de que la actividad productiva del país presenta una alta concentración en la GAM.

Los cuatro conglomerados con una mayor especialización productiva relativa están conformados por cantones de la GAM ,con excepción de Orotina, que es un cantón de la provincia de Alajuela y colindante con la provincia de Puntarenas, el cual podría constituirse como un articulador de desarrollo productivo de la GAM con territorios vecinos o conectados por infraestructura vial, como es el corredor San José-Puerto Caldera.

Por otra parte, la mayoría de los cantones presentan un potencial productivo bajo a nivel de especialización productiva, siendo uno de los ejes que requiere un mayor incentivo para potenciar su desarrollo. Esto es particularmente necesario en territorios fuera de la GAM, que son los que presentan mayor debilidad relativa a los de esa zona, que es donde se han concentrado los incentivos para el desarrollo productivo y la atracción de inversiones.

En cuanto a los conglomerados que se generan en torno al eje infraestructura $y$ al institucional, se repite el patrón de que los cantones con mayor potencial se concentran también dentro de la GAM. No obstante, tanto al Este como al Oeste de esta, se observan cantones con potencial para articular el desarrollo de la GAM con otras zonas del país.

Referente al eje infraestructura, los cantones con mayor potencial productivo relativo se ubican en la GAM y pertenecen a la provincia de Heredia; no obstante, se identifican cantones con potencial relativo tanto en la periferia Este como Oeste de la GAM. Por su parte, los cantones con potencial productivo menor se ubican relativamente lejos de la GAM, concentrándose a lo largo de las costas y fronteras del país.

En el eje infraestructura, se observa una mayor concentración de cantones con potencial productivo hacia la periferia Suroeste de la GAM, cantones que podrían constituirse en importantes núcleos para articular el desarrollo productivo de la GAM con otras regiones y generar mayores niveles de convergencia y expansión del desarrollo productivo.

En particular, en el eje institucional, se identifican casos aislados de cantones con potencial alto en otras regiones del país como es el caso de Hojancha, así como un importante grupo con un potencial medio-alto hacia el Suroeste de la GAM, mayoritariamente pertenecientes a la provincia de Alajuela, que podrían constituirse en núcleos de desarrollo institucional para las regiones aledañas.

Los resultados y hallazgos de este estudio contribuyen para informar el proceso de toma de decisiones en torno al diseño, definición e implementación de políticas de desarrollo productivo con carácter territorial en Costa Rica. El estudio ofrece una serie de criterios técnicos, que han sido empíricamente evaluados $y$ validados, que serán de gran ayuda para identificar y definir territorios con potencial para convertirse en ZEED.

\section{REFERENCIAS}

Arias, R., \& Sánchez, L. (2012). Patrones de concentración y evolución del empleo industrial en la Gran Area Metropilitana (GAM) de Costa Rica. Ciencias Economícas, 30-No 2, 131-154.

Arias, et al. (2015). Aproximación teórica y metodológica para la definición de zona económica especial (ZEE) en Costa Rica. Serie de Divulgación Económica. No. 32, Mayo, IICE-UCR. San José, Costa Rica.

Boix, R. y Galletto, V. (2005). Sistemas Locales de Trabajo y Distritos Industriales Marshallianos en España. Departamento de Economía Aplicada, Universidad Autónoma de Barcelona. Barcelona, España. 
CAATEC (2010). Penetración de Conectividad de Banda Ancha por Cantón. Fundación Comisión Asesora en Alta Tecnología (CAATEC). San José, Costa Rica.

Cabrero, E.; Orihuela, I.; y Ziccardi, A. (2003). "Ciudades competitivas - Ciudades cooperativas: Conceptos claves y construcción de un índice para ciudades mexicanas". Documento de trabajo n ${ }^{\circ}$ 139. División de Administración Pública, CIDE. México.

CCSS (2013). Estadísticas Actuariales: Áreas y Cantones. Caja Costarricense del Seguro Social (CCSS). San José, Costa Rica.

CGR (2011). Índice de Gestión Municipal (IGM 2011). Contraloría General de la Republica de Costa Rica. San José, Costa Rica.

CSE. (2014). Instituciones Parauniversitarias carreras aprobadas, suprimidas y modificaciones curriculares presentadas ante el Consejo Superior de Educación. Consejo Superior de Educación, San José, Costa Rica.

De la Fuente, S. (2011). Análisis Conglomerados. Facultad de Ciencias Económicas y Empresariales, Universidad Autónoma de Madrid (UAM). Madrid, España.

INA (2013). INA en Cifras 2013. Instituto Nacional de Aprendizaje (INA). San José, Costa Rica.

INEC (2011). Censo Nacional de Población. Instituto Nacional de Estadística y Censos (INEC). San José, Costa Rica.

INEC y CCP (2008). Estimaciones y proyecciones de población. Instituto Nacional de Estadística y Censos (INEC) y Centro Centroamericano de Población (CCP) e. San José, Costa Rica.

Ishida, M. (2009). "Special Economic Zones and Economic Corridors" [Zonas Económicas Especiales y corredores económicos]. Development Studies Center, Institute of Developing Economies, Japan, Junio, 1-23.

Kayser, O., Klarsfeld, L., Brossad S. (2014). The Broadband Effect: Enhancing Market-Based solution for the base of the Pyramid. Opportunities for the Majority of the Inter-American Development Bank [El efecto de banda ancha: solución basada en el mercado como generador de la base de la pirámide. Oportunidades para la Mayoría del Banco Interamericano de Desarrollo]. Bank's Documents.

Markussen, A., Hall, P. and Glasmeier, A. (1986). High Tech America: The What, How, Where and Why of the Sunrise Industries[América Alta tecnología: El qué, cómo, y por qué del renacer de las industrias], Boston, MA: Allen \&Unwin.

Millán, F. (1994). "Competitividad internacional de regiones", Serie Ensayos, Instituto Latinoamericano y del Caribe de Planificación Económica y Social (ILPES). Santiago, Chile.

PNUD y UCR (2009). Atlas del desarrollo humano cantonal de Costa Rica. Programa de las Naciones Unidas para el Desarrollo (PNUD) y Universidad de Costa Rica (UCR). San José, Costa Rica.

ProDUS (2013). Presencia de Colegios técnicos. Programa de Investigación en Desarrollo Urbano Sostenible (ProDUS). San José, Costa Rica.

ProDUS (2013). Presencia de instituciones para universitarias. Programa de Investigación en Desarrollo Urbano Sostenible(ProDUS). San José, Costa Rica.

ProDUS (2013). Riesgo ante eventos extremos. Programa de Investigación en Desarrollo Urbano Sostenible (ProDUS). San José, Costa Rica.

ProDUS (2014). Distancia respecto a aeropuertos. Programa de Investigación en Desarrollo Urbano Sostenible (ProDUS). San José, Costa Rica.

ProDUS (2014). Distancia respecto a fronteras. Programa de Investigación en Desarrollo Urbano Sostenible (ProDUS). San José, Costa Rica.

ProDUS (2014). Distancia respecto a puertos. Programa de Investigación en Desarrollo Urbano Sostenible (ProDUS). San José, Costa Rica.

ProDUS (2014). Precio por metro cuadrado. Programa de Investigación en Desarrollo Urbano Sostenible (ProDUS). San José, Costa Rica.

ProDUS (2014). Tenencia y estado del plan regulador. Programa de Investigación en Desarrollo 
Urbano Sostenible (ProDUS). San José, Costa Rica.

Rodríguez, A. (2006). "Desarrollo Económico Territorial: Teorías y aplicación al caso Uruguayo. Instituto de Economía. Junio, Serie: DT 02/06.

Sassen, S. (1991). The Global City: New York, London, Tokyo. Princeton, New Jersey:[La ciudad global: Nueva York, Londres, Tokio. Princeton, Nueva Jersey] Princeton University Press.

TEC (2008). Atlas del TEC. Tecnológico de Costa Rica. Cartago, Costa Rica.

Tong D. Chang Chun, F. GuiCai, L. Peng, C. (2010). "Categories and Characteristics of urban villages by GIS-Based Analysis: A case study of Shenzhen Special Economic Zone"[ Categorías y Características de los pueblos urbanos por Análisis Base-SIG: Un estudio del caso de la Zona Económica Especial de Shenzhen]. Science China technological sciences, May, 53, 150-157.

TSE (2010). Atlas Electoral 2010. Tribunal Supremo de Elecciones (TSE). San José, Costa Rica.

TSE (2014). Atlas Electoral 2014. Dirección General del Registro Electoral y Financiamiento de Partidos Políticos. Tribunal Supremo de Elecciones (TSE). San José, Costa Rica.

Vázquez Barquero, A. (2000). La Política de Desarrollo Económico Local. Marco de Análisis: El Enfoque y la Política. Capítulo 1, 21-45. 
